\title{
Geometry of inter-areal interactions in mouse visual cortex
}

\author{
Ramakrishnan Iyer \\ MindScope Program, Allen Institute, \\ Seattle, WA \\ rami@alleninstitute.org
}

\section{Gayathri Mahalingam}

Allen Institute for Brain Science, Seattle, WA

gayathrim@alleninstitute.org

\author{
Joshua H. Siegle \\ MindScope Program, Allen Institute, \\ Seattle, WA \\ joshs@alleninstitute.org \\ Shawn Olsen \\ MindScope Program, Allen Institute, \\ Seattle, WA \\ shawno@alleninstitute.org
}

\author{
Stefan Mihalas \\ MindScope Program, Allen Institute, \\ Seattle, WA \\ stefanm@alleninstitute.org
}

\begin{abstract}
The response of a set of neurons in an area is the result of the sensory input, the interaction of the neurons within the area as well as the long range interactions between areas. We aimed to study the relation between interactions among multiple areas, and if they are fixed or dynamic. The structural connectivity provides a substrate for these interactions, but anatomical connectivity is not known in sufficient detail and it only gives us a static picture. Using the Allen Brain Observatory Visual Coding Neuropixels dataset, which includes simultaneous recordings of spiking activity from up to 6 hierarchically organized mouse cortical visual areas, we estimate the functional connectivity between neurons using a linear model of responses to flashed static grating stimuli. We characterize functional connectivity between populations via interaction subspaces. We find that distinct subspaces of a source area mediate interactions with distinct target areas, supporting the notion that cortical areas use distinct channels to communicate. Most importantly, using a piecewise linear model for activity within each trial, we find that these interactions evolve dynamically over tens of milliseconds following a stimulus presentation. Inter-areal subspaces become more aligned with the intra-areal subspaces during epochs in which a feedforward wave of activity propagates through visual cortical areas. When the short-term dynamics are averaged over, we find that the interaction subspaces are stable over multiple stimulus blocks. These findings have important implications for understanding how information flows through biological neural networks composed of interconnected modules, each of which may have a distinct functional specialization.
\end{abstract}

\section{Introduction}

The view that the cerebral cortex is organized in multiple areas, each of which may carry out different functions, has been around for more than a century [1]. For the primate [2] as well as for the mouse [3, 4], the visual system is both modular and hierarchical. Different visual areas in mice have 
distinct retinotopic maps [5] and functional specialization [6], but are strongly interconnected with each other [7, 8]. Understanding how different brain areas work together requires observing activity simultaneously across multiple constituent interacting neuronal populations.

The vast majority of our knowledge of cellular-level interactions between cortical regions comes from experiments in which only two areas were observed at a given time. The Allen Brain Observatory Visual Coding Neuropixels dataset includes simultaneous measurements of spiking activity from all layers of 4-6 cortical visual areas, making it possible to address a wide range of questions about the dynamic routing of information. A recent study [9] using this open dataset revealed that the organization of inter-areal functional connectivity during visual stimulation mirrors the structural connectivity hierarchy.

We analyzed this dataset with a view to understand how the different visual areas interact when a simple visual stimulus is presented. Of special importance is the question of the dynamics of these interactions. Our results could help future studies understand the dynamics of the formation of representations across areas when the stimulus is task relevant.

\subsection{Relation to previous work}

Many studies have investigated inter-areal interactions, mostly between two different brain regions. Interactions have been characterized using multiple techniques; a prevalent method consists of recording LFPs in two different areas [10-14]. A corpus of studies focused on understanding the frequency dependence of the signals led to the proposal of communication through coherence [15, 16]. However, it is not yet clear how the oscillations relate to the formation of specific representations of individual cells needed for image-dependent tasks such as object classification. Other methods include the study of pairwise correlations between spiking activity of neurons in different areas [9, 13, 17, 19], or relating spiking activity of a single neuron in one area and a population in another [20, 21]. These have led to important insights into the nature and dependence of interactions on factors such as stimulus drive and task demands.

With the advent of large scale simultaneous recordings from multiple brain regions, studies have begun investigating relationships between multiple neuronal spike trains in different areas using multi-variate statistical methods such as multivariate linear regression, canonical correlation analysis (CCA) and their variants [22-26] (see [27] for a review of methods and related studies). Of particular interest to us is the study by Semedo et al. [23] on interactions between cortical areas V1 and V2 in macaques. Using factor analysis and reduced rank regression for dimensionality reduction, they relate trial-to-trial fluctuations between neuronal populations in these areas to show that V1-V2 interactions are mediated via a communication subspace.

To characterize interactions between six mouse visual cortical areas with a slightly different approach, we used a linear model (with L1-regularization) which predicts target neuron activities using a linear combination of the activities of all simultaneously recorded neurons from multiple brain regions and a stimulus-dependent contribution. Variants of such peer-prediction models have been previously used to characterize interactions within populations [28, 29] and to study inter-areal interactions [20, 21].

Using this model, we obtain interaction matrices between pairs of visual cortical areas. We quantify the degree of alignment between different interaction subspaces using subspace angles. Consistent with the results of [23], we find that intra- and inter-areal interactions are distinct for each visual cortical area in mouse. A unique contribution of our study is the relation between interaction subspaces across multiple visual areas. We find that the inter-areal interactions among visual areas are more aligned than the intra-areal interactions.

We also find that the interaction subspaces are dynamic, with rapid changes over $50 \mathrm{~ms}$ after a stimulus onset. However, when the short-term dynamics are averaged over, the interaction subspaces are stable over stimulus blocks which are $>30$ minutes apart. This creates an interesting comparison with the recent study in [30] which found that attention does not modify the communication subspace. 
a)
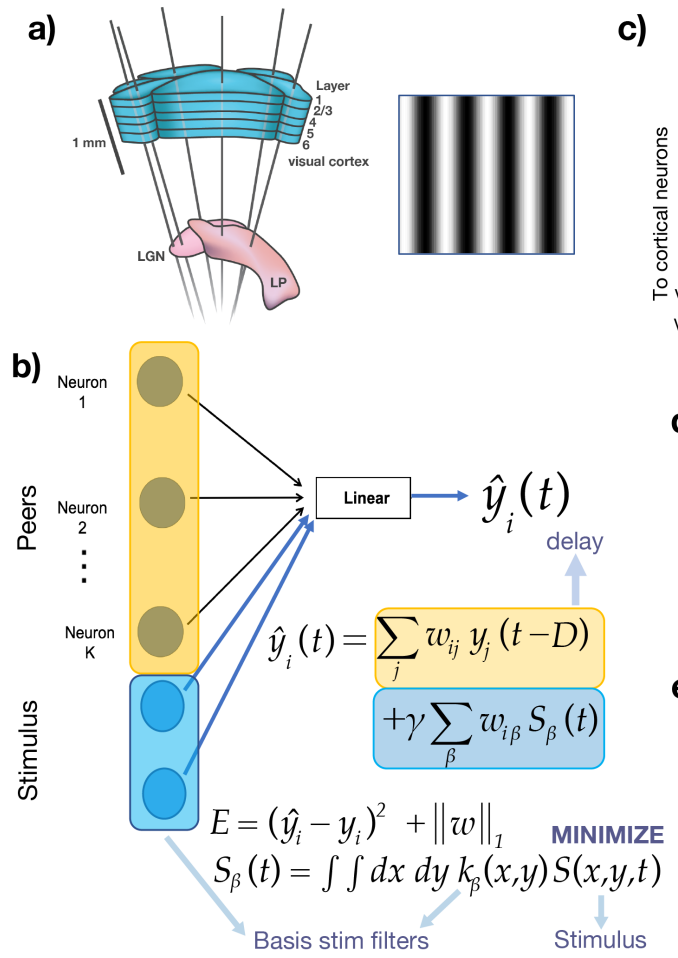

c)

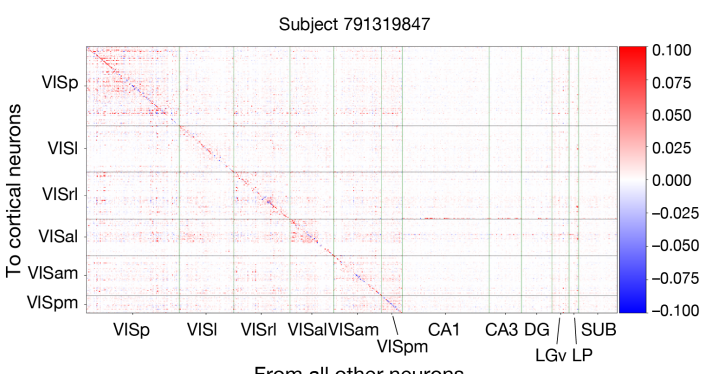

d)

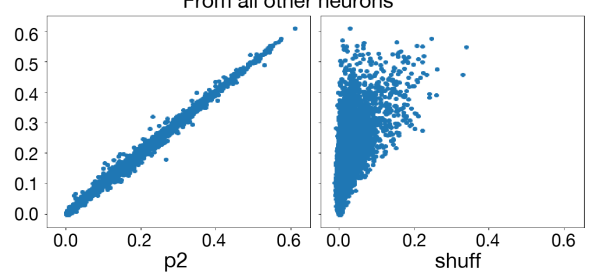

e)

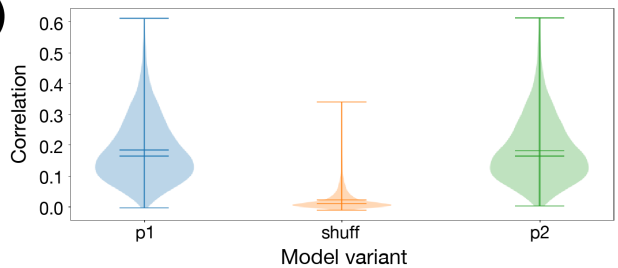

Figure 1: Overview of model for interactions. a) Figure showing six Neuropixels probes targeting cortex and thalamus with static grating stimuli analyzed in this study. b) Schematic of linear model with L1 regularization for single neuron responses using stimulus and peer terms. Stimulus contribution is evaluated using a bank of 120 basis Gabor filters (see Methods). c) Peer coupling coefficients from units in all regions (columns) on to units in all visual cortical regions (rows) from an example session. Vertical and horizontal lines demarcate units within a given source and target region. d) Scatter plot comparing correlations between model predictions and test data for all visual cortical units $(\approx 5700$ units $)$ for three model variants. In all cases, the vertical axis represents test correlations from the model trained on the first half of data $(\mathrm{p} 1)$. The horizontal axis shows correlation from model variants. Left : model trained on the second half of data (p2) and tested against the first half; Right: model trained using shuffled data (see Methods). e) Distribution of correlations between model predictions and test data for all units in six visual cortical regions for the three model variants.

\section{Results}

\subsection{Peer prediction model captures interactions between neurons in different brain regions}

We used the Allen Brain Observatory Visual Coding Neuropixels dataset (https://portal.brainmap.org/explore/circuits/visual-coding-neuropixels). It contains simultaneous recordings of neuronal responses to a variety of visual stimuli from six visual cortical areas (VISp, VISl, VISal, VISrl, VISpm and VISam) in addition to thalamic regions LP, dLGN and hippocampus in awake head-fixed mice on a wheel using Neuropixels probes. Further details can be found in [9].

We analyzed responses to flashed static grating stimuli (Fig. 1a). We focus on the flashed gratings as they are relatively simple stimuli which still allow us to reveal a temporal dependence of interactions. Recent work [31] has shown that functional interactions can be more easily discovered by analyzing stimulus-driven responses of the network than just from spontaneous activity. The stimulus set comprised of gratings with six orientations $(0,30,60,90,120,150)$ degrees, five spatial frequencies $(0.02,0.04,0.08,0.16,0.32) \mathrm{cpd}$ and four phases $(0,45,90,135)$ degrees. Each grating was flashed for $250 \mathrm{~ms}$ approximately 50 times for a total of 25 minutes of static grating stimulus presentations (with gray screens of the same duration randomly interleaved approximately every 30 presentations). Stimuli were presented in three epochs of 9,8 and 8 minutes respectively with 22 and 15.5 minutes gaps between the blocks. We selected neurons based on three quality metrics which ensured that 
units were relatively stable across the entirety of each session $(\approx 90$ minutes $)$ and had low levels of spike train contamination (see Methods). We chose 19 sessions that contained at least 10 units in five out of six visual cortical areas. This gave us a total of 5686 units across the 19 chosen sessions. We provide a breakdown of the number of units per visual area for each of the 19 sessions analyzed in (Supplement: Experimental data). We collected together spike-times for each neuron in a session across all three epochs and binned them in $10 \mathrm{~ms}$ bins.

To investigate the nature of interactions between mouse visual cortical areas, we developed a generalized linear model to predict single neuron activity on each trial of stimulus presentation (Fig. 1b, see Methods). Figure 1c shows a matrix of coupling coefficients from all neurons to all visual cortical neurons estimated from the model for an example session. While individual neurons' own spiking history provides some of the largest coefficients as seen from the diagonal terms in the matrix, the model also captures strong long-range influences from neurons in multiple other brain regions. Most of the largest coefficients come from other visual cortical and thalamic regions which are well-known to be involved in visual stimulus processing. We also show coefficients from stimulus contributions in (Supplement: Model for interactions).

We constructed different model variants labeled (p1, p2, shuff) to ascertain the robustness of our estimates for the coupling coefficients and subsequent analyses. These models differ in the training data that is used to estimate the weights (see Methods). We evaluated model performance using the correlation between model prediction and test data. Figure 1d shows scatter plots of correlations between the base model (p1) and model variants (p2; left plot) and (shuff; right plot) for all 5686 visual cortical neurons analyzed. The left plot shows that the model is robust to choice of training data and is not sensitive to the particular stimulus epochs chosen to train the model. The right plot shows that the base model outperforms the shuffled model indicating that it is capable of capturing meaningful stimulus-specific patterns of interactions. Correlations range from slightly negative to as high as 0.6 for a few neurons, with medians $0.163(0.163)$ respectively for $\mathrm{p} 1$ (p2) and 0.009 for shuffled data, comparable to values reported in [32]. Figure 1e shows distributions of correlations for the three model variants. Neurons having high correlation even with shuffled data are likely high-firing neurons (possibly interneurons) that are agnostic to stimulus.

\subsection{Cortical areas interact via distinct subspaces}

It was shown in [23] that cortical areas V1 and V2 in macaques interact via a communication subspace. V2 activity was related to a small subset of population activity patterns in V1 and these patterns were distinct from shared V1 fluctuations which were most predictive of V1 activity. Motivated by their findings, we used the estimated functional interaction matrices from our model to investigate how distinct are interactions between pairs of mouse visual cortical areas. Figure 2a shows a schematic of this idea.

To probe the geometry of functional interactions between pairs of visual cortical areas, we calculated the subspace angles $\theta\left(S, T_{1}, T_{2}\right)$ between subspaces of estimated interaction matrices $\left(M_{1}, M_{2}\right)$ from a source area $S$ to pairs of target areas $\left(T_{1}, T_{2}\right)$ (see Methods). The subspace angle generalizes the notion of angle between a vector and 2D plane in 3 dimensions. It measures the degree of alignment between two subspaces in a higher dimensional space and varies from 0 degrees (indicating perfect alignment) to 90 degrees (indicating orthogonality between the two subspaces). Intuitively, the subspace angles capture the angles between the first few most important modes of interaction between $M_{1}$ and $M_{2}$.

We first verified that the subspace angles obtained from our model provide a robust measure of interaction geometry by comparing the angles between matrices for a source and target area pair obtained from model variants ( $1, \mathrm{p} 2)$ containing recordings from different stimulus blocks for both original and shuffled data. Figure $2 \mathrm{~b}$ shows angles between matrices from source $S$ (VISp here) to all visual cortical areas averaged across all sessions. Figure $2 \mathrm{c}$ shows a scatter plot comparing first (top row) and second (bottom row) angles from original and shuffled data for all possible pairs of source and target areas across all sessions, both for intra-areal (blue) and inter-areal (orange) interactions. The angles from shuffled data are significantly higher than those from original data coming from different stimulus blocks. This tells us that the change in the interaction subspaces across stimulus blocks together with the intrinsic variability in estimating the subsapces is small compared to the suffle condition. It is interesting to note here that the first angles from original and shuffled data are 

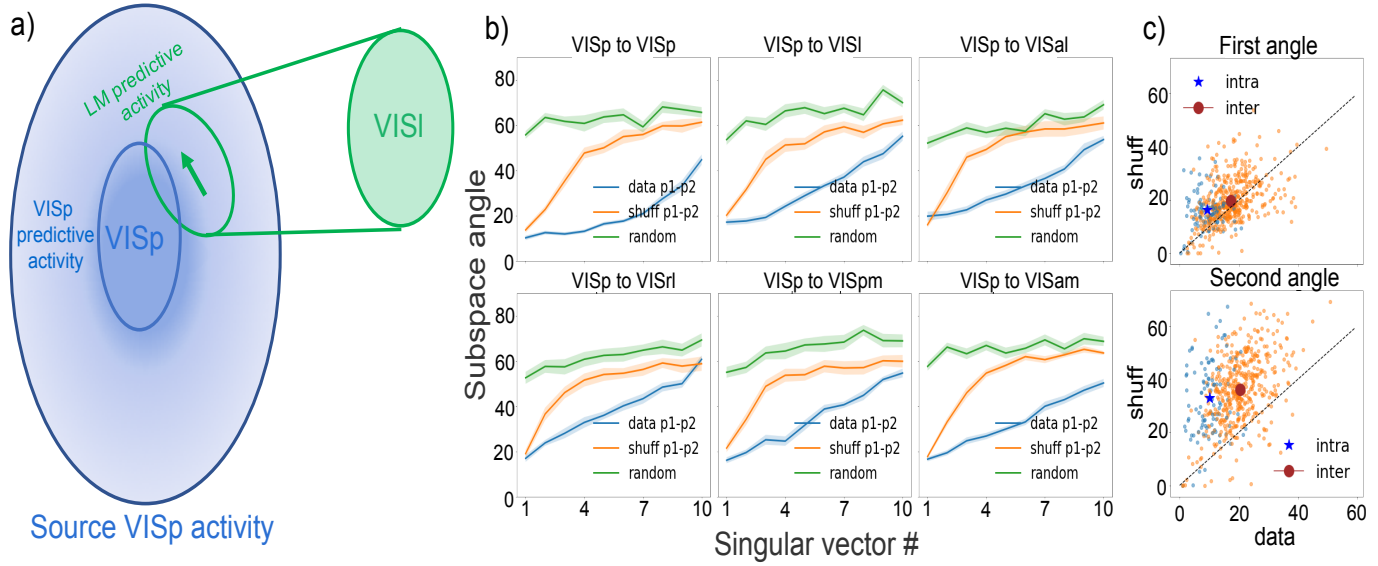

d)
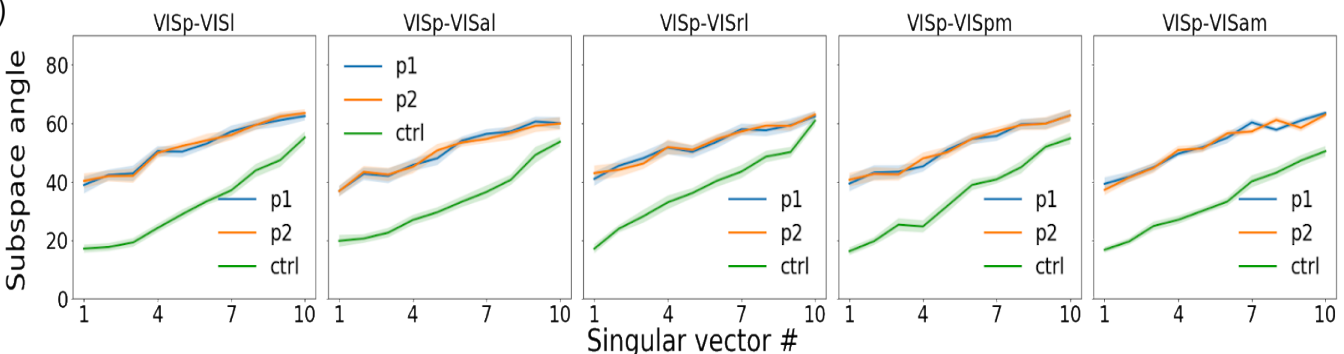

Figure 2: Distinct subspaces of source areas mediate intra- and inter-areal interactions. a) Schematic of the idea showing that distinct subspaces in VISp mediate interactions within VISp and outside with VISl. b) Subspace angles (see Methods) provide a measure of how distinct interaction subspaces between pairs of areas are aligned. Subspace angles for interaction matrices from VISp to six target regions estimated using first and second halves of activity for original data (blue), shuffled data (orange) and a fully randomized condition (see Methods). Solid lines represent session averages; shaded regions show std error of mean (sem). c) Scatter plots of first (top) and second (bottom) subspace angles from original data (horizontal axis) vs shuffled data (vertical axis) for intra-areal (blue) and inter-areal (orange) interactions. Angles from original data are significantly lower than those obtained using shuffled data (Wilcoxon signed-rank test; pvals : $\left(1.8 \times 10^{-13}, 3.7 \times 10^{-9}, 4.4 \times 10^{-18}, 7.3 \times 10^{-67}\right)$ respectively for first intra-, first inter-, second intra- and second inter-areal interaction angles.) d) Subspace angles from a fixed source region (VISp shown here) to (VISp, T) where T is one of five higher visual areas [T : VIS1, VISal, VISrl, VISpm, VISam] for model variants p1 (blue) and p2 (orange). Angles are significantly higher than inter-areal interaction angles (green) estimated from activities in different stimulus blocks (blue lines in Fig. 2b) as well the shuffle controls (see Supplement: Cortical areas interact via distinct subspaces).

quite close to each other. This is likely due to the fact that in both cases, the binned activities have been aligned to stimulus onset.

Next, we investigated the subspace angles between intra-areal and inter-areal interactions - namely recurrent interactions between neurons within a source area and the interactions of the same group of neurons with those in other areas. Using the notation introduced above, we are interested in the angles $\theta(S, S, T)$ when $M_{1}$ represents recurrent interactions within $S$ and $M_{2}$ represents interactions between $S$ and an external target area $T$.

The angles between these subspaces are significantly higher than the angles between inter-areal subspaces estimated for different stimulus blocks (Fig 2d) and they are also significantly higher than shuffle control (Supplement: Cortical areas interact via distinct subspaces). These results are consistent with the findings in macaque for V1-V2 interactions [23] and extend beyond VISp (V1) to all higher visual cortical areas in mouse. We also computed subspace angles after estimating interaction matrices using the approach of [23]. We show in (Supplement: Cortical areas interact via distinct subspaces) that this latter method produces qualitatively similar results.

We then asked how aligned are subspace interactions from a source cortical area to two external cortical areas. Are external-external interactions from a source population of neurons mediated via distinct modes (Fig. 3a)? We are thus interested in the cases when $M_{1}$ and $M_{2}$ correspond to 


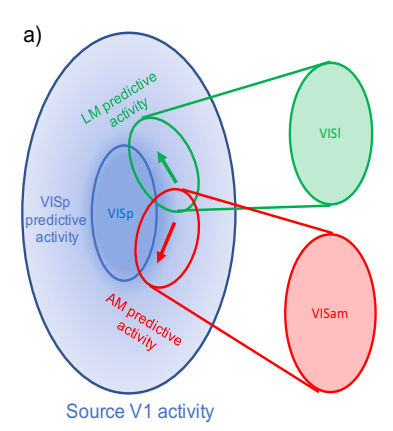

Schematic of the idea
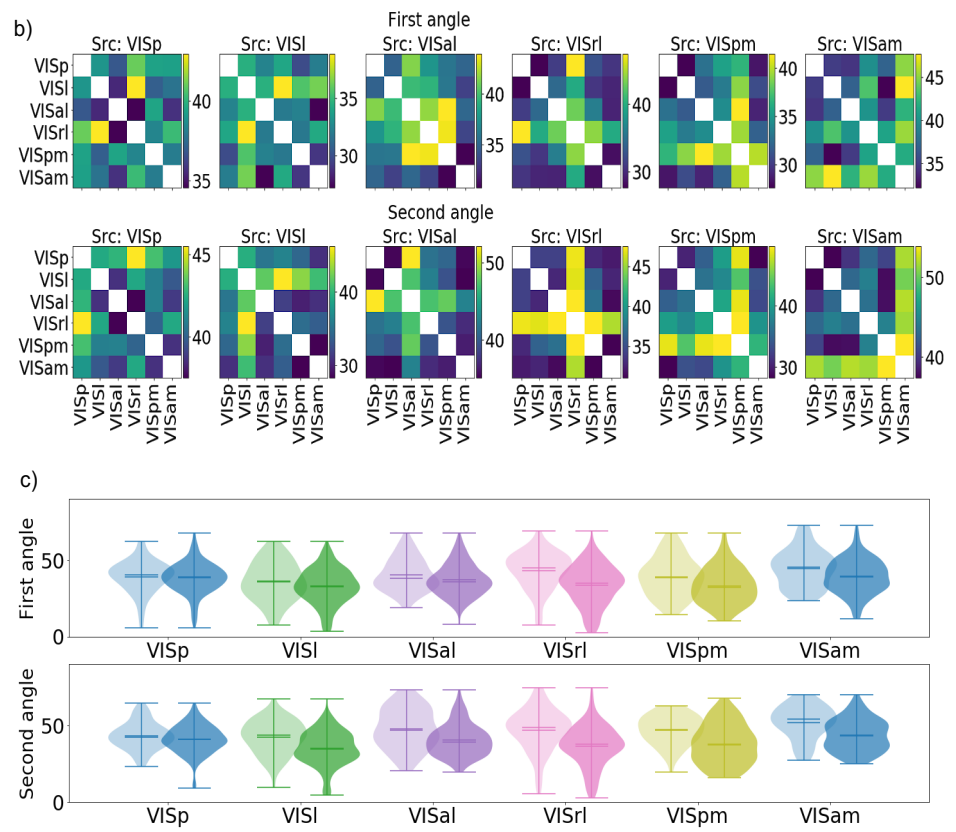

Figure 3: Internal-external interaction subspaces are less aligned than external-external interaction subspaces. a) Schematic of the idea showing that distinct subspaces in VISp mediate interactions with external targets VISl and VISam. b) Heatmaps of first (top row) and second (bottom row) subspace angles between all possible subspaces within each source cortical area. Each pixel represents the subspace angle between $\left(S, T_{1}\right)$ and $\left(S, T_{2}\right)$ where $S$ is the source area and each (row,column) corresponds to $\left(T_{1}, T_{2}\right)$. The heatmaps are symmetric corresponding to exchange of the $\left(T_{1}, T_{2}\right)$ labels. Note that the colorbars are on different scales. c) Distribution of internal-external and external-external angles (top; first angle, bottom; second angle). Each pair of violinplots with different shades of the same color represents internal-external (light shade) and external-external (dark shade) angles respectively.

interactions between source area $S$ and external target areas $T_{1}$ and $T_{2}$. We show in (Supplement: Cortical areas interact via distinct subspaces) that angles $\theta\left(S, T_{1}, T_{2}\right)$ between external-external interactions are again higher than shuffle controls.

\subsection{Internal-external interaction subspaces are less aligned than external-external interaction subspaces}

Having established that cortical areas interact via distinct subspaces, we next investigated if there are differences between internal-external interactions and external-external interactions from a source area to different target areas. Specifically, we compared the subspace angles $\theta\left(S, S, T_{1}\right)$ and $\theta\left(S, T_{1}, T_{2}\right)$. We use internal to refer to recurrent interactions within an area and external to refer to interactions from a source area to an external target.

Figure $3 \mathrm{~b}$ shows the first (top) and second (bottom) subspace angles from a source area to pairs of target areas averaged across all sessions. For each of the six visual cortical areas, the angles between internal-external interaction subspaces are higher than the angles between external-external interaction subspaces. This is particularly prominent when the source area is VISrl - an area that is known to be weakly responsive to visual stimuli compared with other visual cortical areas and has strong connections to somatosensory areas [8].

Figure $3 \mathrm{e}$ shows the comparison between distributions of the internal-external and external-external first (top) and second (bottom) angles from all sessions. For each pair of distributions (except for the first angle for source VISp), the internal-external angles are significantly higher than the externalexternal angles (Benjamini-Hochberg FDR-corrected p-values for the Mann-Whitney U test for six cortical areas respectively: $\left(0.128,0.031,0.021,6.69 \times 10^{-8}, 0.001,0.003\right)$ for the first angles and $\left(0.031,6.56 \times 10^{-7}, 1.04 \times 10^{-5}, 2.53 \times 10^{-9}, 1.82 \times 10^{-7}, 8.76 \times 10^{-8}\right)$ for the second angles $)$. 
Do similar patterns of interactions exist within task-trained artificial neural networks? We have shown that intra-areal interaction subspaces are not well aligned with the inter-areal interaction subspaces (Figure 3). To allow for such a possibility, the network is likely to require interactions between neurons within an area. Therefore we analyzed interactions between the representation modules in PredNet [33, 34] - a deep convolutional recurrent neural network that was trained for next-frame video prediction. PredNet's architecture was inspired by principles of predictive coding from neuroscience [35], but it includes both feedback from higher areas as well as lateral connection on top of the feedforward connections.

We provide detailed results of our analysis in (Supplement: PredNet analysis). Briefly, we 'probed' the activations of a subset of neurons within representation layers $R_{1}, R_{3}$ and $R_{3}$ in PredNet in response to video sequences. These are the layers that have explicit recurrent connections. We used the peer coupling model to estimate internal-external and external-external interaction subspace angles between these modules. In contrast to the biological network, our analysis failed to reveal significant differences between internal-external and external-external interactions within PredNet.

\subsection{Subspaces exhibit greater alignment during the wave of activity elicited by flashed stimuli}

Next we investigated if the degree of alignment between interaction subspaces changes dynamically over the course of tens of milliseconds within a single trial of stimulus presentation. We divided activities evoked over the course of a $250 \mathrm{~ms}$ stimulus presentation into five successive epochs of 50 $\mathrm{ms}$ each from the time of stimulus onset (Fig. 4a top). Using the peer-prediction model, we estimated interaction matrices between cortical areas within each $50 \mathrm{~ms}$ epoch and computed the subspace angles from a source area to all possible pairs of target areas. Our analysis reveals that rather than being static, the degree of alignment between interaction subspaces changes within a single trial of stimulus presentation.

Heatmaps in Fig 4a middle and bottom rows respectively show the first and second subspace angles from a source area (VISl shown here) to a pair of target cortical areas for each $50 \mathrm{~ms}$ epoch. It can be seen that the angle between interaction subspaces becomes smaller during the $(50-100 \mathrm{~ms})$ and $(100-150 \mathrm{~ms})$ epochs before gradually becoming similar to the $(0-50 \mathrm{~ms})$ epoch. This holds true for each of the six visual cortical areas (Supplement: Subspace alignment within trials).

For both internal-external and external-interactions, the angles during the (50-100ms) and (100$150 \mathrm{~ms})$ epochs are significantly smaller than the angle during the $(0-50 \mathrm{~ms})$ epoch ((BenjaminiHochberg FDR-corrected p-values for Wilcoxon signed-rank test comparing average angles for epochs 2-5 with epoch 1; first angle p-values : $\left(1.00 \times 10^{-9}, 1.00 \times 10^{-9}, 5.69 \times 10^{-4}, 3.15 \times 10^{-2}\right)$, second angle p-values : $\left.\left(2.65 \times 10^{-12}, 1.27 \times 10^{-13}, 1.82 \times 10^{-1}, 1.66 \times 10^{-4}\right)\right)$. Although the angles decrease overall, the internal-external angles still remain higher than the external-external angles within each epoch. This can be seen in the first columns of Fig.4b,c which respectively show the first and second angles averaged across all sessions and areas for internal-external (blue) and external-external (orange) interactions.

Session-averaged first and second angles for each source visual cortical area are shown respectively in Fig. 4b,c middle column (internal-external angles) and last column (external-external angles). These reveal a rich variety of dynamics depending on the specific source area. For example, VISam, which is at the top of the visual hierarchy [4, 9] does not show the same decrease in intra- and inter-areal interaction subspace angles as areas lower in the hierarchy. VISrl angles rebound faster than the other areas, decreasing during the $50-100 \mathrm{~ms}$ epoch and increasing again during the $100-150 \mathrm{~ms}$ epoch.

One important question is if this observed change is the result of changes in firing rates within these intervals. To address this, we used a stimulus shuffle (see Methods and Figure 2, model variant shuff). The same shuffle, which preserves the temporal structure of the average firing rate of the neurons was used, but it was applied to neuronal activities separated into $50 \mathrm{~ms}$ time intervals. We applied the same method to compute the subspace angles and their relative alignment for the shuffled data, and we subtracted the shuffle control from all the angles computed. We find that the results are unchanged when the shuffled angles are subtracted, with significant alignment between the inter- and intra-areal subspaces 50-150 ms after the stimulus onset compared to 0-50ms (see Supplement: Cortical areas interact via distinct subspaces). 
bioRxiv preprint doi: https://doi.org/10.1101/2021.06.09.447638; this version posted June 23, 2021. The copyright holder for this preprint (which was not certified by peer review) is the author/funder, who has granted bioRxiv a license to display the preprint in perpetuity. It is made available under aCC-BY-NC-ND 4.0 International license.

a)
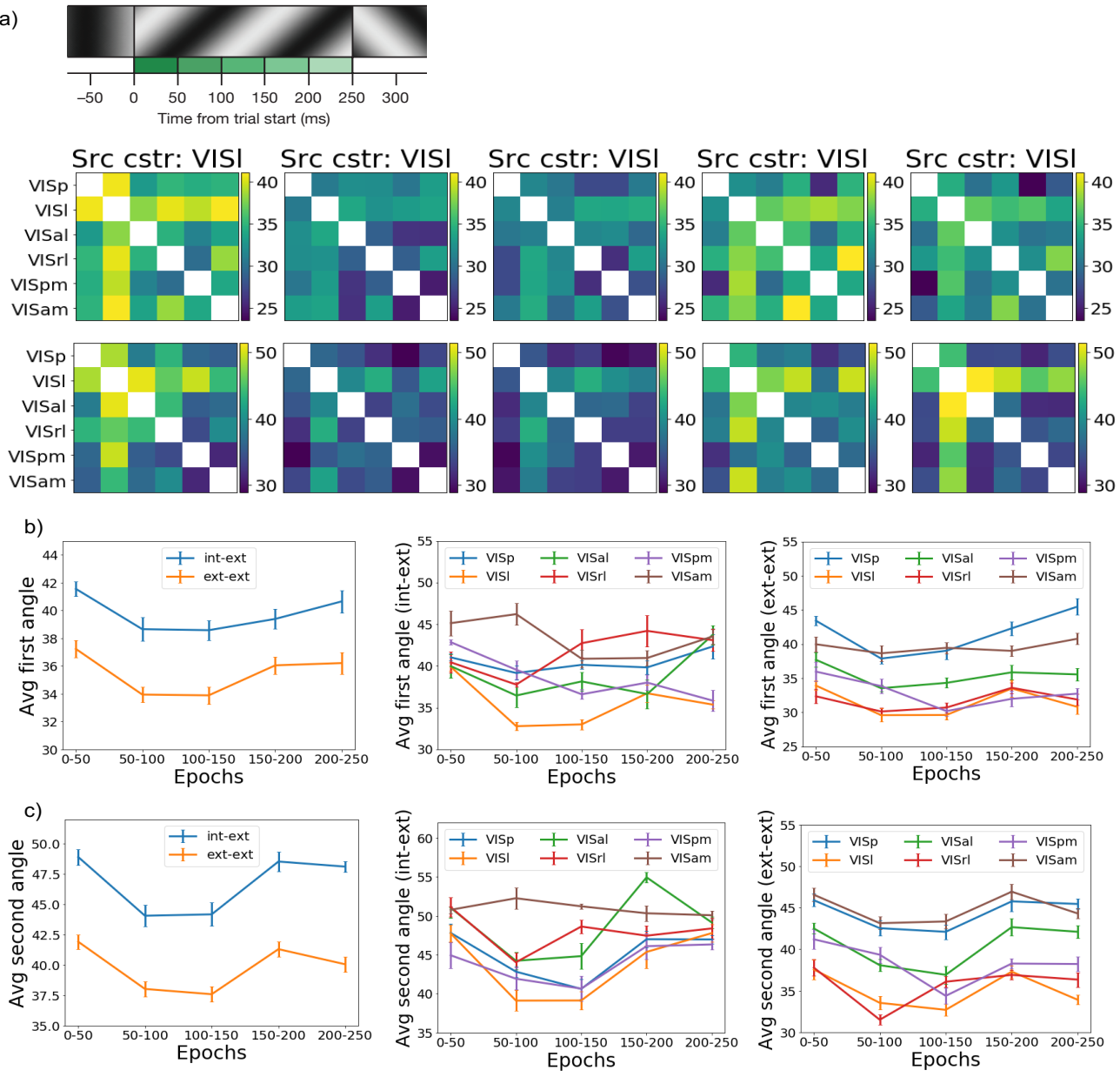

Figure 4: Subspaces rotate within single trials and become more aligned after stimulus onset. a) Top: Schematic showing division of $50 \mathrm{~ms}$ epochs within a single trial from stimulus onset. Bottom first row: Heatmap of first angle from a source cortical area (VISl shown here) to all possible target cortical area pairs for five successive $50 \mathrm{~ms}$ epochs (columns) within $250 \mathrm{~ms}$ trials. Bottom second row: Second angles. Angles were computed by estimating interaction matrices using activity within each $50 \mathrm{~ms}$ epoch. Note that although subspaces become more aligned, internal-external subspace pairs are still more orthogonal than external-external subspace pairs within each epoch. b) left) Average of all combinations of internal-external (blue) and external-external (orange) first angles for the five $50 \mathrm{~ms}$ epochs showing subspace alignment between 50-150 ms after stimulus onset, middle) average of all internal-external first angles across five $50 \mathrm{~ms}$ epochs separated by source cortical area and right) average of all external-external first angles across five $50 \mathrm{~ms}$ epochs separated by source cortical area. c) Same as in b) but for second angles. 


\section{Discussion}

Our study confirms the distinction between the intra- and inter-areal communication subspaces characterized by Semedo et al. [23] in the visual cortex of a different model species (mouse vs primate) and with a slightly different method (see (Supplement: Cortical areas interact via distinct subspaces) for a more direct comparison). Our study's contributions are that: 1 . We observe that the distinction between intra- and inter-areal interaction subspaces generalizes across all six visual cortical areas, and that the subspaces are stable across stimulus blocks. 2. While all the subspaces are distinct, the intra-areal interaction subspaces are significantly less aligned than the inter-areal interaction subspaces. 3. Most importantly, we observe that the interaction subspaces are dynamic, and can rotate within 50ms following a stimulus onset. However, when averaging over the short-time dynamics, the interaction subspaces are stable over stimulus blocks $>30$ minutes apart. The latter finding can be compared with [30] which found that attention does not modify the communication subspace.

There are multiple limitations of our study. While it would be most interesting to analyze the dynamics of subspaces across task conditions, the available data is limited to passive viewing. It would be interesting to compare the dynamics of interaction subspaces with other methods (e.g. communication by coherence). Another limitation is that we characterized the interaction subspaces only among visual areas, and interactions with other modules/non-visual areas might be different. The biggest limitation is that we do not have a mechanistic understanding of the circuit structure which causes such a misalignment of inter- and intra-areal subspaces, or the mechanism which causes them to rotate and be better aligned following the stimulus onset. We hoped to be able to peek inside the black box by using an existing artificial neural network and performing experiments by modifying its connectivity structure and seeing when different features of the biologically observed code are lost. However, we could not run such analyses since the network we analyzed (PredNet) did not reproduce the main features observed in biology to begin with.

One way to interpret our results is that in biological networks, individual areas have inter-areal dynamics which is not well aligned with the intra-areal dynamics, but this alignment becomes better for a short period of time following a stimulus onset (i.e. cortical areas communicate what they are doing to other areas only when needed). This communication principle seems different from one artificial recurrent neural network that we looked at. Our study opens up questions about the importance of such a communication principle in more complex tasks in biological and artificial neural networks.

\section{Methods}

\subsection{Experimental methods overview}

Experimental methods are described in detail in [9]. Mice were head-fixed with the right eye placed 15 $\mathrm{cm}$ from a visual stimulus monitor, and were free to run on a rotating disk throughout the experiment. Neuropixels probes [36] were inserted into each cortical area, as well as underlying subcortical regions, such as hippocampus and thalamus. Data was acquired with the Open Ephys GUI [37], and spikes were extracted offline using Kilosort2 [38]. Units found by Kilosort2 were selected for further analysis based on three quality metrics thresholds: presence ratio $>0.95$, ISI violations $<0.5$, amplitude cutoff $<0.1$ (see [9] for definitions). In total, we analyzed 5686 neurons from 19 mice.

\subsection{Peer prediction model for interactions}

The instantaneous firing rate $y_{i}(t)$ of an individual neuron was predicted in time-bins of size $10 \mathrm{~ms}$ as the weighted sum of a peer-prediction term and a stimulus-dependent term as follows,

$$
\hat{y}_{i}(t)=\sum_{j} w_{i j} y_{j}(t-D)+\gamma \sum_{\beta} w_{i \beta} \int d x \int d y k_{\beta}(x, y) S(x, y, t)
$$

where $k_{\beta}(x, y)$ represents a basis set of 120 gabor filters and $S(x, y, t)$ represents the static grating stimulus. Gabor filters were parameterized using static grating parameters (6 orientations, 5 spatial frequencies, 4 phases) used in the experiments and $D$ represents a delay that allows us to include the spiking history of individual neurons. We chose $D=10 \mathrm{~ms}$ for our model. 
Coefficients $w_{i j}$ and $w_{i \beta}$ were fit using linear regression with an $L_{1}$ regularization term $\alpha_{\gamma}\|w\|_{1}$. The regularization term constrains the number of non-zero coefficients in the model and explains away contributions from neurons which may themselves be highly correlated. $\gamma$ controls the relative contributions of peer activity and stimulus. For a fixed value of $\gamma$, the hyper-parameter $\alpha_{\gamma}$ was optimized for via 10-fold cross validation on training data. $\gamma$ itself was varied on a grid with $\gamma=$ $(0.0,0.01,0.5,0.1,1.0,5.0,10.0,50.0)$ and was chosen to be the one that maximized the correlation between model prediction and test data. We used scikit-learn's LassoCV method for fitting. Having done this for a subset of the 19 sessions, we noticed that the specific choice of $\gamma$ did not lead to significant differences in the correlation between model prediction and test data. So without loss of generality, we fixed $\gamma=1$ for all results reported here.

\subsubsection{Model variants}

We constructed different model variants to ascertain the robustness of estimates for coupling coefficients and subsequent analyses. We refer to these model variants as (p1, p2, shuff, random) respectively.The base model (p1) used first half of observed spiking activity for estimating weights and evaluated model performance on the remaining half, model (p2) used the second half of spiking activity to estimate model weights and evaluate performance on the first half, model (shuff) used a shuffled version of binned activity in which the stimulus labels were independently permuted for each neuron while retaining the within trial bins and model (random) used a shuffled version of binned activity in which both stimulus and within trial bins were independently permuted for each neuron.

\subsection{Computation of subspace angles}

For every pair $\left(M_{1}, M_{2}\right)$ of interaction matrices between pairs of visual cortical areas, we quantified the degree of alignment between corresponding low-dimensional subspaces using subspace angles (also called principal angles between subspaces). Principal angles $\theta_{i}(i=1,2, \cdots, q)$ between two matrices $U_{n \times p}$ and $V_{n \times q}$ with $p>q$ are defined [39, 40] by $\cos \theta_{k}=\max _{u \in U} \max _{v \in V} u_{k}^{T} v_{k}$. To standardize for differences in numbers of recorded neurons between source and target (and thereby the dimensionality of the estimated interaction matrices), we first used singular value decomposition (SVD) to reconstruct 10-dimensional subspaces $\left(M_{1}^{\prime}, M_{2}^{\prime}\right)$ using the first 10 respective singular vectors. We then calculated the angles between each singular vector of respectively $M_{1}\left(M_{2}\right)$ and the corresponding 10-dimensional subspace $M_{2}^{\prime}\left(M_{1}^{\prime}\right)$ respectively. The average of these two angles was used to quantify the subspace angle between $M_{1}$ and $M_{2}$. Directly computing the subspace angles between $\left(M_{1}^{\prime}, M_{2}^{\prime}\right)$ did not affect our conclusions (Supplement: Computation of subspace angles). Subspace angles have been used in [41] to show that OFF responses in auditory cortex to different stimuli lie mostly in orthogonal subspaces.

\section{Acknowledgments and Disclosure of Funding}

We wish to thank the Allen Institute founder, Paul G. Allen, for his vision, encouragement and support.

\section{References}

[1] Korbinian Brodmann. Vergleichende Lokalisationslehre der Grosshirnrinde in ihren Prinzipien dargestellt auf Grund des Zellenbaues. Barth, 1909.

[2] Daniel J Felleman and David C Van Essen. Distributed hierarchical processing in the primate cerebral cortex. Cerebral cortex (New York, NY: 1991), 1(1):1-47, 1991.

[3] Quanxin Wang, Olaf Sporns, and Andreas Burkhalter. Network analysis of corticocortical connections reveals ventral and dorsal processing streams in mouse visual cortex. Journal of Neuroscience, 32(13):4386-4399, 2012.

[4] Julie A Harris, Stefan Mihalas, Karla E Hirokawa, Jennifer D Whitesell, Hannah Choi, Amy Bernard, Phillip Bohn, Shiella Caldejon, Linzy Casal, Andrew Cho, et al. Hierarchical organization of cortical and thalamic connectivity. Nature, 575(7781):195-202, 2019. 
[5] Jun Zhuang, Lydia Ng, Derric Williams, Matthew Valley, Yang Li, Marina Garrett, and Jack Waters. An extended retinotopic map of mouse cortex. Elife, 6:e18372, 2017.

[6] James H Marshel, Marina E Garrett, Ian Nauhaus, and Edward M Callaway. Functional specialization of seven mouse visual cortical areas. Neuron, 72(6):1040-1054, 2011.

[7] Seung Wook Oh, Julie A Harris, Lydia Ng, Brent Winslow, Nicholas Cain, Stefan Mihalas, Quanxin Wang, Chris Lau, Leonard Kuan, Alex M Henry, et al. A mesoscale connectome of the mouse brain. Nature, 508(7495):207-214, 2014.

[8] Joseph E Knox, Kameron Decker Harris, Nile Graddis, Jennifer D Whitesell, Hongkui Zeng, Julie A Harris, Eric Shea-Brown, and Stefan Mihalas. High-resolution data-driven model of the mouse connectome. Network Neuroscience, 3(1):217-236, 2018.

[9] Joshua H Siegle, Xiaoxuan Jia, Séverine Durand, Sam Gale, Corbett Bennett, Nile Graddis, Greggory Heller, Tamina K Ramirez, Hannah Choi, Jennifer A Luviano, et al. Survey of spiking in the mouse visual system reveals functional hierarchy. Nature, 592(7852):86-92, 2021.

[10] Georgia G Gregoriou, Stephen J Gotts, Huihui Zhou, and Robert Desimone. Highfrequency, long-range coupling between prefrontal and visual cortex during attention. science, 324(5931):1207-1210, 2009.

[11] RF Salazar, NM Dotson, SL Bressler, and CM Gray. Content-specific fronto-parietal synchronization during visual working memory. Science, 338(6110):1097-1100, 2012.

[12] Conrado A Bosman, Jan-Mathijs Schoffelen, Nicolas Brunet, Robert Oostenveld, Andre M Bastos, Thilo Womelsdorf, Birthe Rubehn, Thomas Stieglitz, Peter De Weerd, and Pascal Fries. Attentional stimulus selection through selective synchronization between monkey visual areas. Neuron, 75(5):875-888, 2012.

[13] Xiaoxuan Jia, Seiji Tanabe, and Adam Kohn. Gamma and the coordination of spiking activity in early visual cortex. Neuron, 77(4):762-774, 2013.

[14] Andre Moraes Bastos, Julien Vezoli, Conrado Arturo Bosman, Jan-Mathijs Schoffelen, Robert Oostenveld, Jarrod Robert Dowdall, Peter De Weerd, Henry Kennedy, and Pascal Fries. Visual areas exert feedforward and feedback influences through distinct frequency channels. Neuron, 85(2):390-401, 2015.

[15] Pascal Fries. Rhythms for cognition: communication through coherence. Neuron, 88(1):220$235,2015$.

[16] Adam Kohn, Anna I Jasper, João D Semedo, Evren Gokcen, Christian K Machens, and M Yu Byron. Principles of corticocortical communication: proposed schemes and design considerations. Trends in Neurosciences, 2020.

[17] R Clay Reid and Jose-Manuel Alonso. Specificity of monosynaptic connections from thalamus to visual cortex. Nature, 378(6554):281-284, 1995.

[18] LG Nowak, MHJ Munk, AC James, Pascal Girard, and J Bullier. Cross-correlation study of the temporal interactions between areas v1 and v2 of the macaque monkey. Journal of Neurophysiology, 81(3):1057-1074, 1999.

[19] Douglas A Ruff and Marlene R Cohen. Attention increases spike count correlations between visual cortical areas. Journal of Neuroscience, 36(28):7523-7534, 2016.

[20] Wilson Truccolo, Leigh R Hochberg, and John P Donoghue. Collective dynamics in human and monkey sensorimotor cortex: predicting single neuron spikes. Nature neuroscience, 13(1):105$111,2010$.

[21] Amin Zandvakili and Adam Kohn. Coordinated neuronal activity enhances corticocortical communication. Neuron, 87(4):827-839, 2015.

[22] Matthew T Kaufman, Mark M Churchland, Stephen I Ryu, and Krishna V Shenoy. Cortical activity in the null space: permitting preparation without movement. Nature neuroscience, 17(3):440-448, 2014. 
[23] João D Semedo, Amin Zandvakili, Christian K Machens, M Yu Byron, and Adam Kohn. Cortical areas interact through a communication subspace. Neuron, 102(1):249-259, 2019.

[24] Douglas A Ruff and Marlene R Cohen. Simultaneous multi-area recordings suggest that attention improves performance by reshaping stimulus representations. Nature neuroscience, 22(10):1669-1676, 2019.

[25] Katherine Cora Ames and Mark M Churchland. Motor cortex signals for each arm are mixed across hemispheres and neurons yet partitioned within the population response. Elife, 8:e46159, 2019.

[26] TL Veuthey, K Derosier, S Kondapavulur, and K Ganguly. Single-trial cross-area neural population dynamics during long-term skill learning. Nature communications, 11(1):1-15, 2020.

[27] João D Semedo, Evren Gokcen, Christian K Machens, Adam Kohn, and M Yu Byron. Statistical methods for dissecting interactions between brain areas. Current Opinion in Neurobiology, 65:59-69, 2020.

[28] Kenneth D Harris, Jozsef Csicsvari, Hajime Hirase, George Dragoi, and György Buzsáki. Organization of cell assemblies in the hippocampus. Nature, 424(6948):552-556, 2003.

[29] Jonathan W Pillow, Jonathon Shlens, Liam Paninski, Alexander Sher, Alan M Litke, EJ Chichilnisky, and Eero P Simoncelli. Spatio-temporal correlations and visual signalling in a complete neuronal population. Nature, 454(7207):995-999, 2008.

[30] Ramanujan Srinath, Douglas A Ruff, and Marlene R Cohen. Attention improves information flow between neuronal populations without changing the communication subspace. bioRxiv, 2021.

[31] Abhranil Das and Ila R Fiete. Systematic errors in connectivity inferred from activity in strongly recurrent networks. Nature Neuroscience, 23(10):1286-1296, 2020.

[32] Saskia EJ de Vries, Jerome A Lecoq, Michael A Buice, Peter A Groblewski, Gabriel K Ocker, Michael Oliver, David Feng, Nicholas Cain, Peter Ledochowitsch, Daniel Millman, et al. A large-scale standardized physiological survey reveals functional organization of the mouse visual cortex. Nature neuroscience, 23(1):138-151, 2020.

[33] William Lotter, Gabriel Kreiman, and David Cox. Deep predictive coding networks for video prediction and unsupervised learning. arXiv preprint arXiv:1605.08104, 2016.

[34] William Lotter, Gabriel Kreiman, and David Cox. A neural network trained for prediction mimics diverse features of biological neurons and perception. Nature Machine Intelligence, 2(4):210-219, 2020.

[35] Rajesh PN Rao and Dana H Ballard. Predictive coding in the visual cortex: a functional interpretation of some extra-classical receptive-field effects. Nature neuroscience, 2(1):79-87, 1999.

[36] James J Jun, Nicholas A Steinmetz, Joshua H Siegle, Daniel J Denman, Marius Bauza, Brian Barbarits, Albert K Lee, Costas A Anastassiou, Alexandru Andrei, Çağatay Aydın, et al. Fully integrated silicon probes for high-density recording of neural activity. Nature, 551(7679):232236, 2017.

[37] Joshua H Siegle, Aarón Cuevas López, Yogi A Patel, Kirill Abramov, Shay Ohayon, and Jakob Voigts. Open ephys: an open-source, plugin-based platform for multichannel electrophysiology. Journal of neural engineering, 14(4):045003, 2017.

[38] Carsen Stringer, Marius Pachitariu, Nicholas Steinmetz, Charu Bai Reddy, Matteo Carandini, and Kenneth D Harris. Spontaneous behaviors drive multidimensional, brainwide activity. Science, 364(6437), 2019.

[39] ke Björck and Gene H Golub. Numerical methods for computing angles between linear subspaces. Mathematics of computation, 27(123):579-594, 1973. 
[40] Andrew V Knyazev and Merico E Argentati. Principal angles between subspaces in an a-based scalar product: algorithms and perturbation estimates. SIAM Journal on Scientific Computing, 23(6):2008-2040, 2002.

[41] Giulio Bondanelli, Thomas Deneux, Brice Bathellier, and Srdjan Ostojic. Network dynamics underlying off responses in the auditory cortex. Elife, 10:e53151, 2021. 


\section{Supplement: Geometry of inter-areal interactions in mouse visual cortex}

\section{S1 Experimental data}

We selected neurons based on three quality metrics which ensured that units were relatively stable across the entirety of each session $(\approx 90$ minutes) and had low levels of spike train contamination (see Methods). We chose 19 sessions that contained at least 10 units in five out of six visual cortical areas. This gave us a total of 5686 units across the 19 chosen sessions. We provide a breakdown of the number of units per visual area for each of the 19 sessions analyzed in Figure S1]

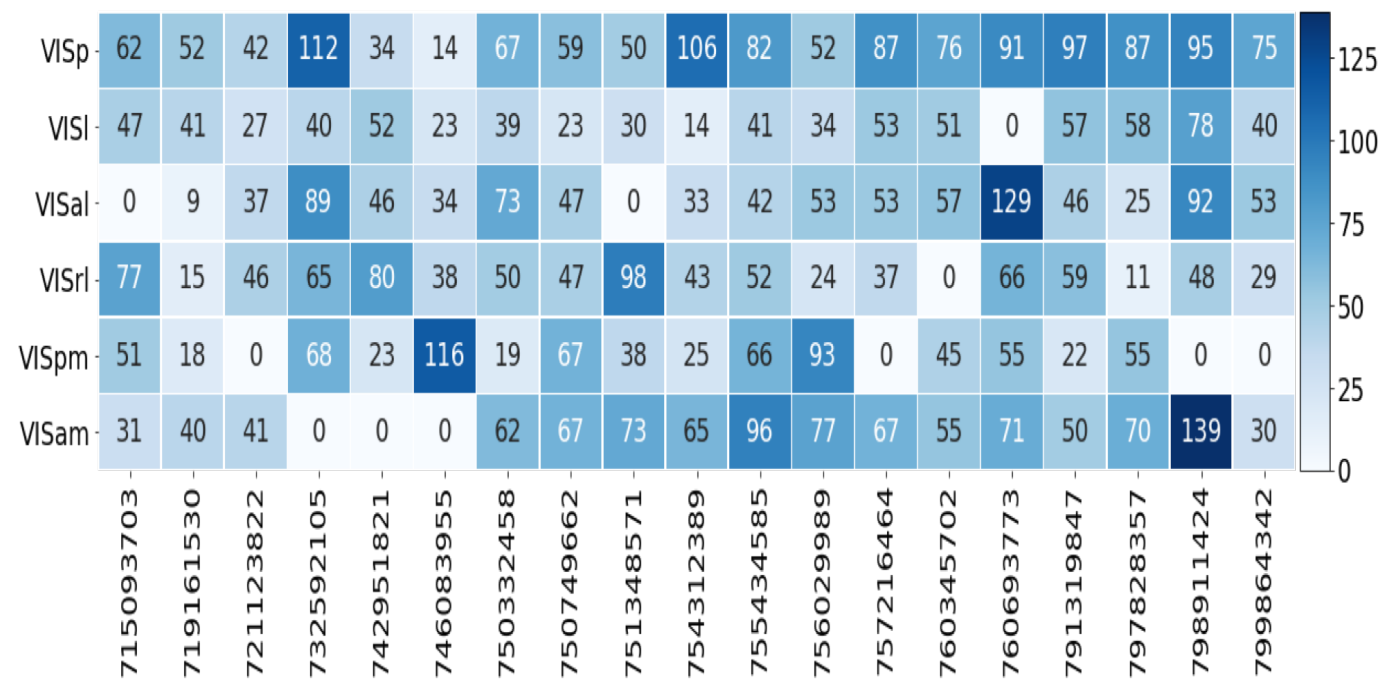

Figure S1: Number of units per visual area for each session analyzed after checking quality metrics (see Methods).

\section{S2 Model for interactions}

In our model, the instantaneous firing rate $y_{i}(t)$ of an individual neuron was predicted in time-bins of size $10 \mathrm{~ms}$ as the weighted sum of a peer-prediction term and a stimulus-dependent term as follows,

$$
\hat{y}_{i}(t)=\sum_{j} w_{i j} y_{j}(t-D)+\gamma \sum_{\beta} w_{i \beta} \int d x \int d y k_{\beta}(x, y) S(x, y, t)
$$

where $k_{\beta}(x, y)$ represents a basis set of 120 gabor filters and $S(x, y, t)$ represents the static grating stimulus. Gabor filters were parameterized using static grating parameters (6 orientations, 5 spatial frequencies, 4 phases) used in the experiments and $D$ represents a delay that allows us to include the spiking history of individual neurons. We chose $D=10 \mathrm{~ms}$ for our model.

Figure $\mathbf{S 2}$ shows a matrix of coefficients $w_{i \beta}$ from the 120 basis gabor filters on to all visual cortical units from an example session.

To standardize for differences in numbers of recorded neurons between source and target (and thereby the dimensionality of the estimated interaction matrices) before computing subspace angles, we first used singular value decomposition (SVD) to reconstruct 10-dimensional subspaces $\left(M_{1}^{\prime}, M_{2}^{\prime}\right)$ using the first 10 respective singular vectors. Figure S3 shows a plot of the cumulative variance explained in an interaction matrix as a function of the number of singular vectors for VISp-VISp, VISp-VISI and VISp-VISam interactions averaged over all 19 sessions. We are able to capture atleast $75 \%$ of the variance in these interaction matrices with $N=10$ singular vectors. 


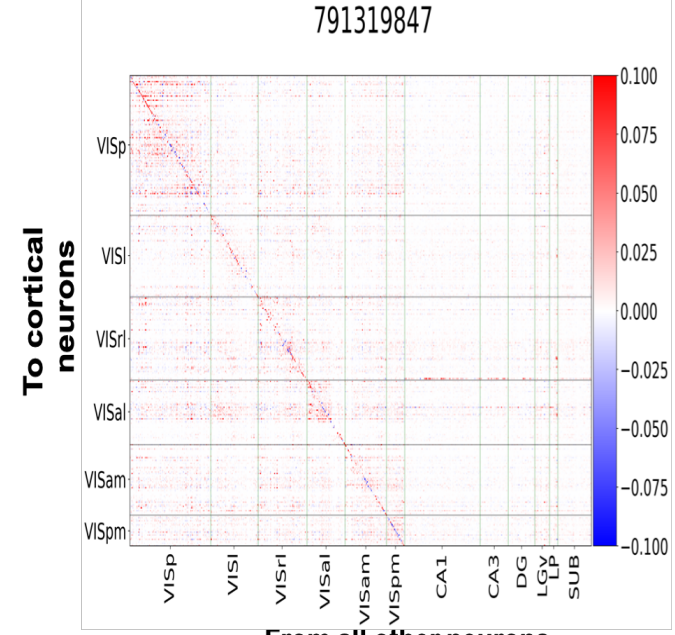

From all other neurons

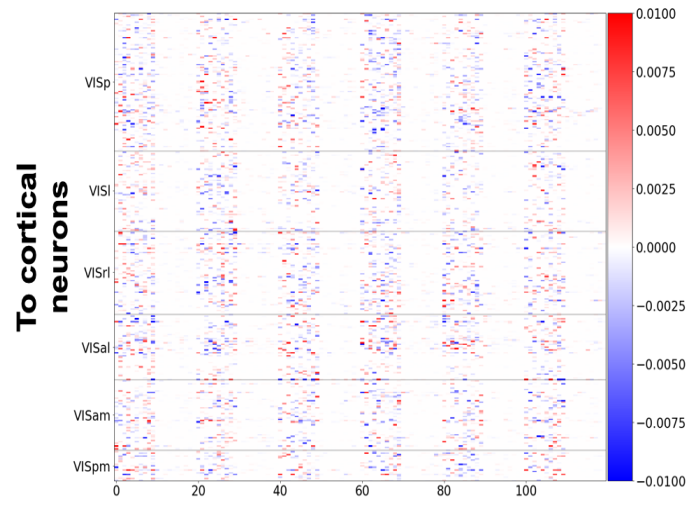

From basis gabor filters

Figure S2: Coefficients for peer coupling and stimulus contribution for one example session. Left: Peer coupling coefficients from units in all regions (columns) on to units in all visual cortical regions (rows). Vertical and horizontal lines demarcate units within a given source and target region. Right: Stimulus coupling coefficients from bank of 120 basis gabor filters (columns) on to units in all visual cortical regions (rows). Horizontal lines demarcate units within a given target region. Colorbar scale has been adjusted for clarity. Each block of 20 columns corresponds to one orientation and each such block is sorted by spatial frequency from low to high. Lower spatial frequencies lead to larger coefficients in the model.

a)

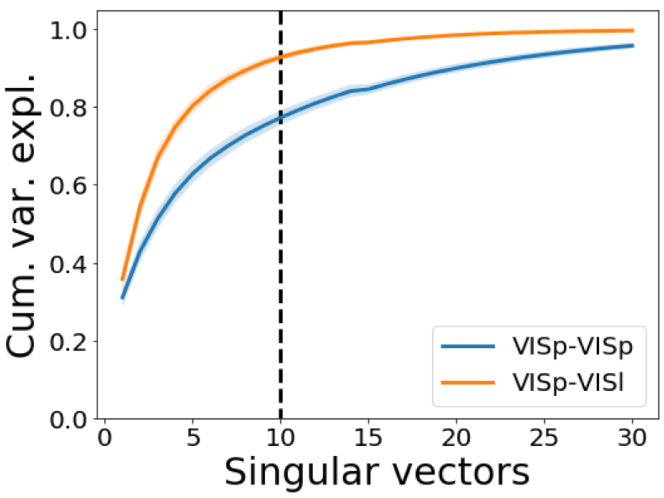

b)

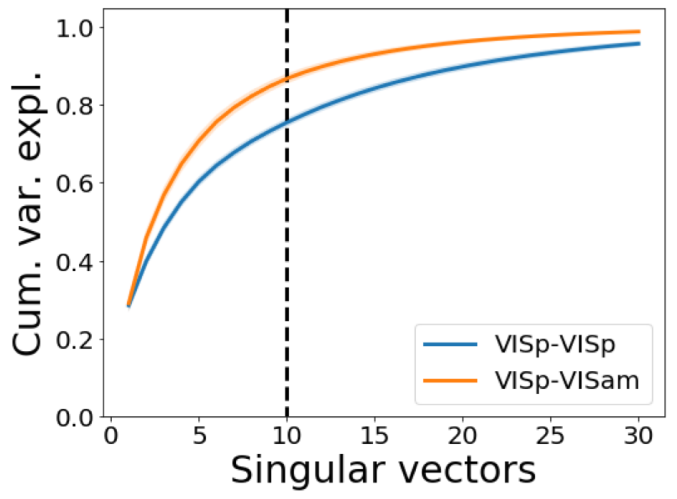

Figure S3: Energy/cumulative variance explained with increasing number of singular vectors of interaction matrices averaged across all sessions. a) Interaction matrices VISp-VISp (blue) and VISp-VISl (orange), b) Interaction matrices VISp-VISp (blue; same curve as in a) and VISp-VISam (orange). Vertical dashed lines correspond to number of singular vectors we used for estimating subspace angles. Our choice of $N=10$ singular vectors captures at least $75 \%$ of variance in the interaction matrices.

\section{S2.1 Total amount of compute and type of resources}

Models parameters for each unit were estimated with parallelization using nodes on our internal cluster. For each unit, model fitting took $\approx 5$ minutes on average and used 10GB of memory. Across all analyses and model variants presented, we had to run approximately 100,000 jobs.

\section{S3 Cortical areas interact via distinct subspaces}

Figure $\mathrm{S} 4$ shows that internal-external first and second angles $\theta(S, S, T)$ are significantly higher than those obtained after shuffling the activities (see Methods, model variant shuff. Wilcoxon signed-rank test pvals $\left(9.0 \times 10^{-15}, 5.6 \times 10^{-12}, 2.8 \times 10^{-13}, 3.9 \times 10^{-14}, 1.5 \times 10^{-11}, 5.6 \times 10^{-13}\right)$ 
respectively for first angles in Fig. S4e top row and $\left(3.9 \times 10^{-11}, 5.2 \times 10^{-8}, 2.6 \times 10^{-7}, 0.0001,0.002,5.3 \times 10^{-6}\right)$ for second angles seen in Fig.S4e bottom row for six source visual cortical areas respectively).
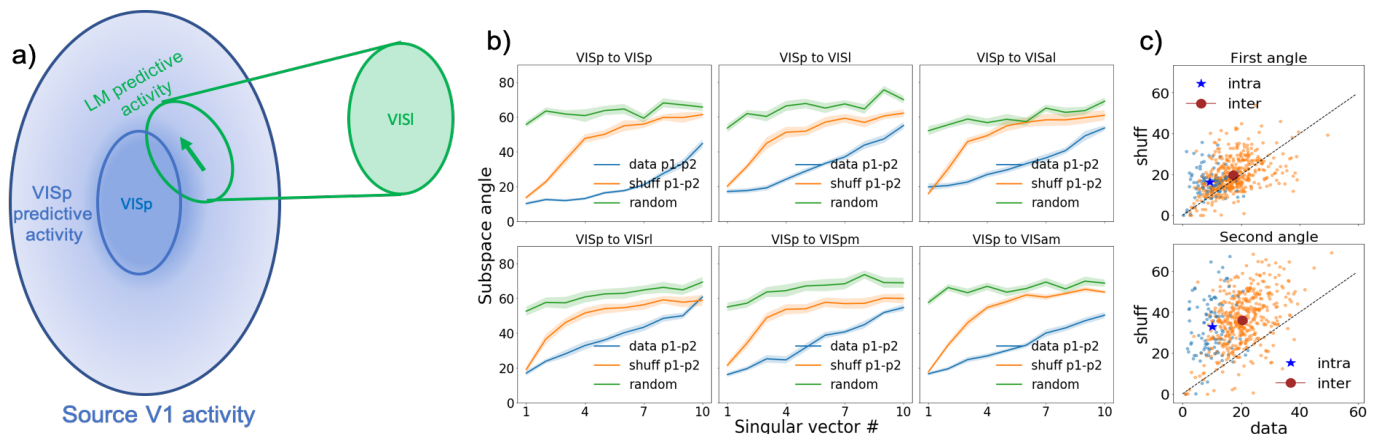

d)
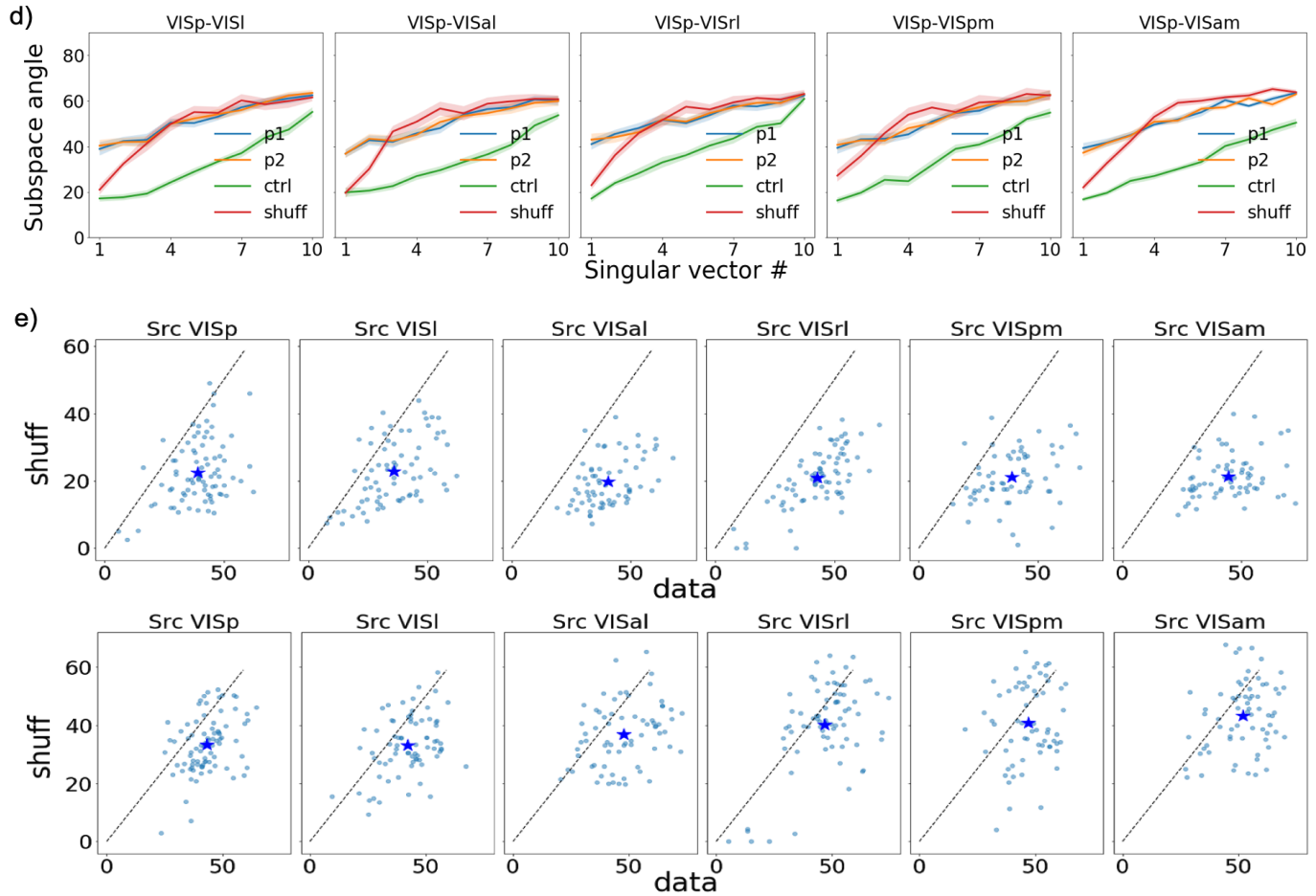

Figure S4: Distinct subspaces of source areas mediate intra- and inter-areal interactions. a) Schematic of the idea showing that distinct subspaces in VISp mediate interactions within VISp and outside with VISl. b) Subspace angles (see Methods) provide a measure of how distinct interaction subspaces between pairs of areas are aligned. Subspace angles for interaction matrices from VISp to six target regions estimated using first and second halves of activity for original data (blue), shuffled data (orange) and a fully randomized condition (see Methods). Solid lines represent session averages; shaded regions show std error of mean (sem). c) Scatter plots of first (top) and second (bottom) subspace angles from original data (horizontal axis) vs shuffled data (vertical axis) for intra-areal (blue) and inter-areal (orange) interactions. Angles from original data are significantly lower than those obtained using shuffled data (Wilcoxon signed-rank test; pvals : $\left(1.8 \times 10^{-13}, 3.7 \times 10^{-9}, 4.4 \times 10^{-18}, 7.3 \times 10^{-67}\right)$ respectively for first intra-, first inter-, second intra- and second inter-areal interaction angles.) d) Subspace angles from a fixed source region (VISp shown here) to (VISp, T) where $\mathrm{T}$ is one of five higher visual areas [T : VISl, VISal, VISrl, VISpm, VISam] for model variants p1 (blue) and p2 (orange). First few angles are significantly higher than inter-areal interaction angles (green) estimated from activities in different stimulus blocks (blue lines in Fig. 2b) as well the shuffle control (red). e,f) Scatter plots of first and second subspace angles separately for each session and source-target combination from original data (horizontal axis) vs shuffled data (vertical axis). The markers show the mean subspace angles.

Angles $\theta\left(S, T_{1}, T_{2}\right)$ between external-external interactions are also higher than shuffle controls for the first angle as can be seen in Figure S5 ((Wilcoxon signed-rank test; pvals : 
bioRxiv preprint doi: https://doi.org/10.1101/2021.06.09.447638; this version posted June 23, 2021. The copyright holder for this preprint (which was not certified by peer review) is the author/funder, who has granted bioRxiv a license to display the preprint in perpetuity. It is made available under aCC-BY-NC-ND 4.0 International license.

$\left(1.9 \times 10^{-38}, 6.3 \times 10^{-22}, 1.2 \times 10^{-31}, 1.3 \times 10^{-31}, 3.7 \times 10^{-25}, 1.9 \times 10^{-36}\right)$ respectively for first angles (Fig. S5c)).

However, the second angles in this case are not significantly higher than shuffle controls except for source area VISp.((Wilcoxon signed-rank test; pvals : $(0.003,0.997,0.878,0.999,0.999,0.999)$ for second angles (Fig. S5d) for six source visual cortical areas respectively).

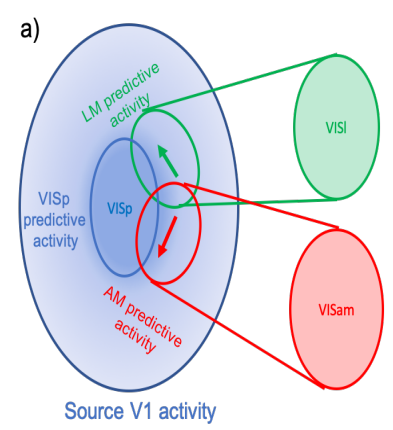

Schematic of the idea
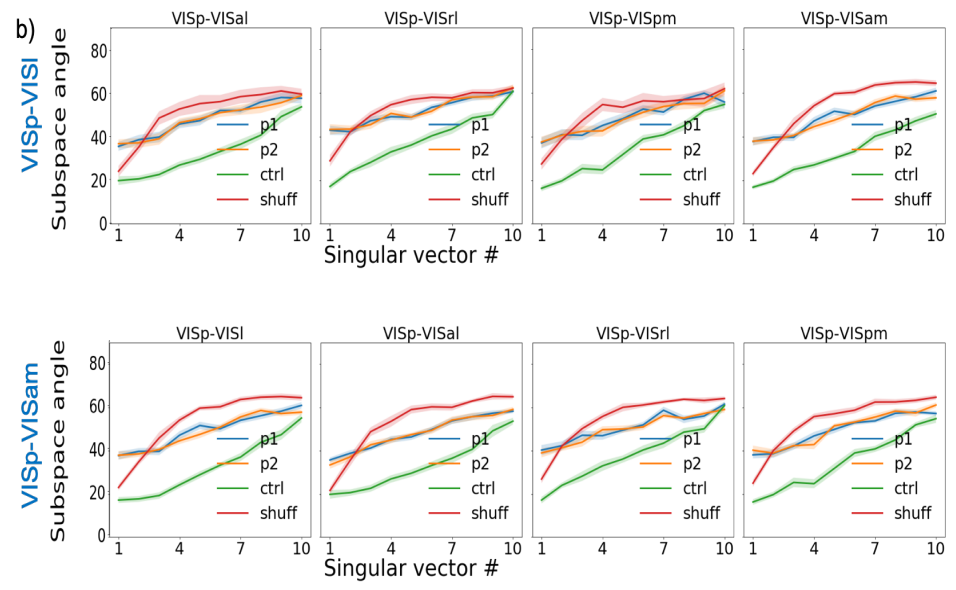

c)
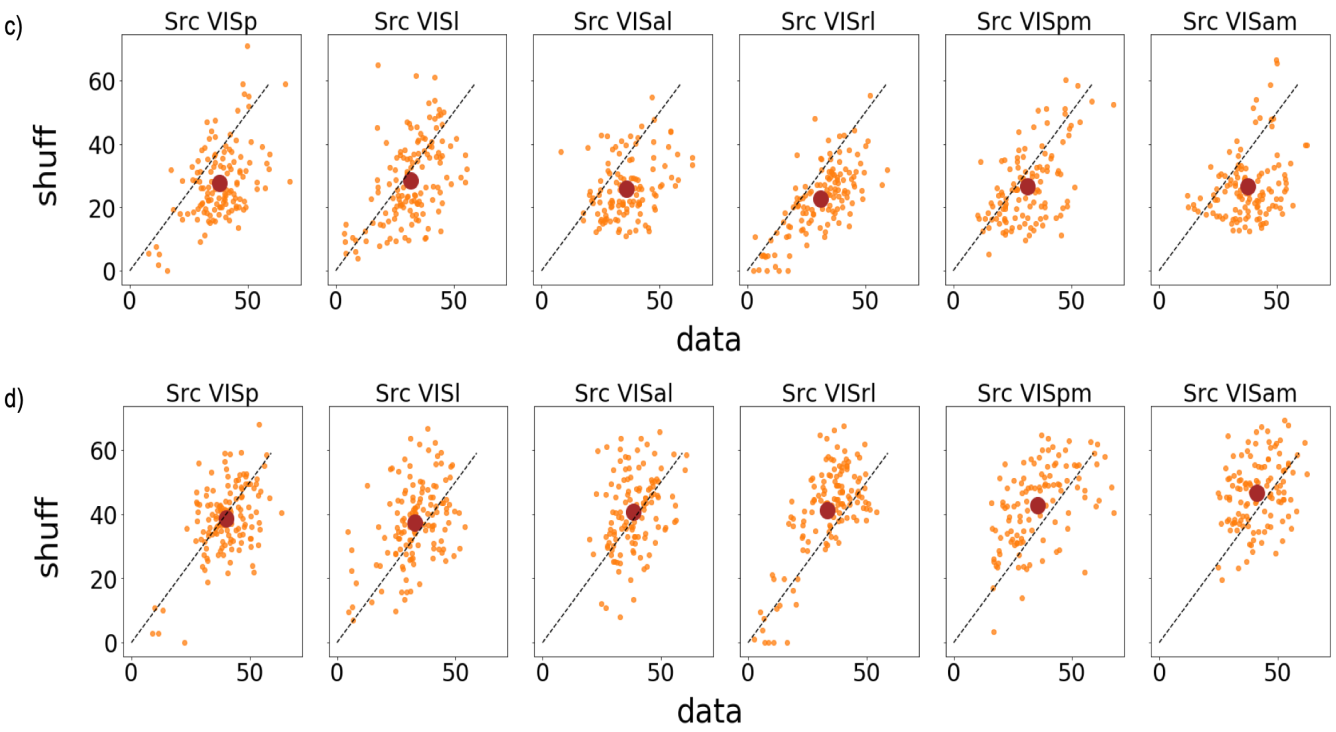

Figure S5: Distinct subspaces of source areas mediate interactions with external target areas. a) Schematic of the idea showing that distinct subspaces in VISp mediate interactions outside with VISl and VISam. b) Subspace angles between VISp-VISl (top) and VISp-VISam (bottom) subspaces and remaining four external target subspaces estimated using first (blue) and second (orange) halves of activity for original data, shuffled data (red) and angles (green) estimated from activities in different stimulus blocks (blue lines in Fig. 2b and Fig. S4b. Solid lines represent session averages; shaded regions show std error of mean (sem). c, d) Scatter plots of first and second subspace angles separately for each session and source-target combination from original data (horizontal axis) vs shuffled data (vertical axis). The markers show the mean subspace angles.

\section{S3.1 Subspaces exhibit greater alignment during the wave of activity elicited by flashed stimuli}

We have shown (Figure 4) that subspaces exhibit greater alignment during the wave of activity elicited by flashed stimuli between $50-150 \mathrm{~ms}$ after stimulus onset. An important question is if this observed change is the result of changes in firing rates within these intervals. To address this, we used 
a)
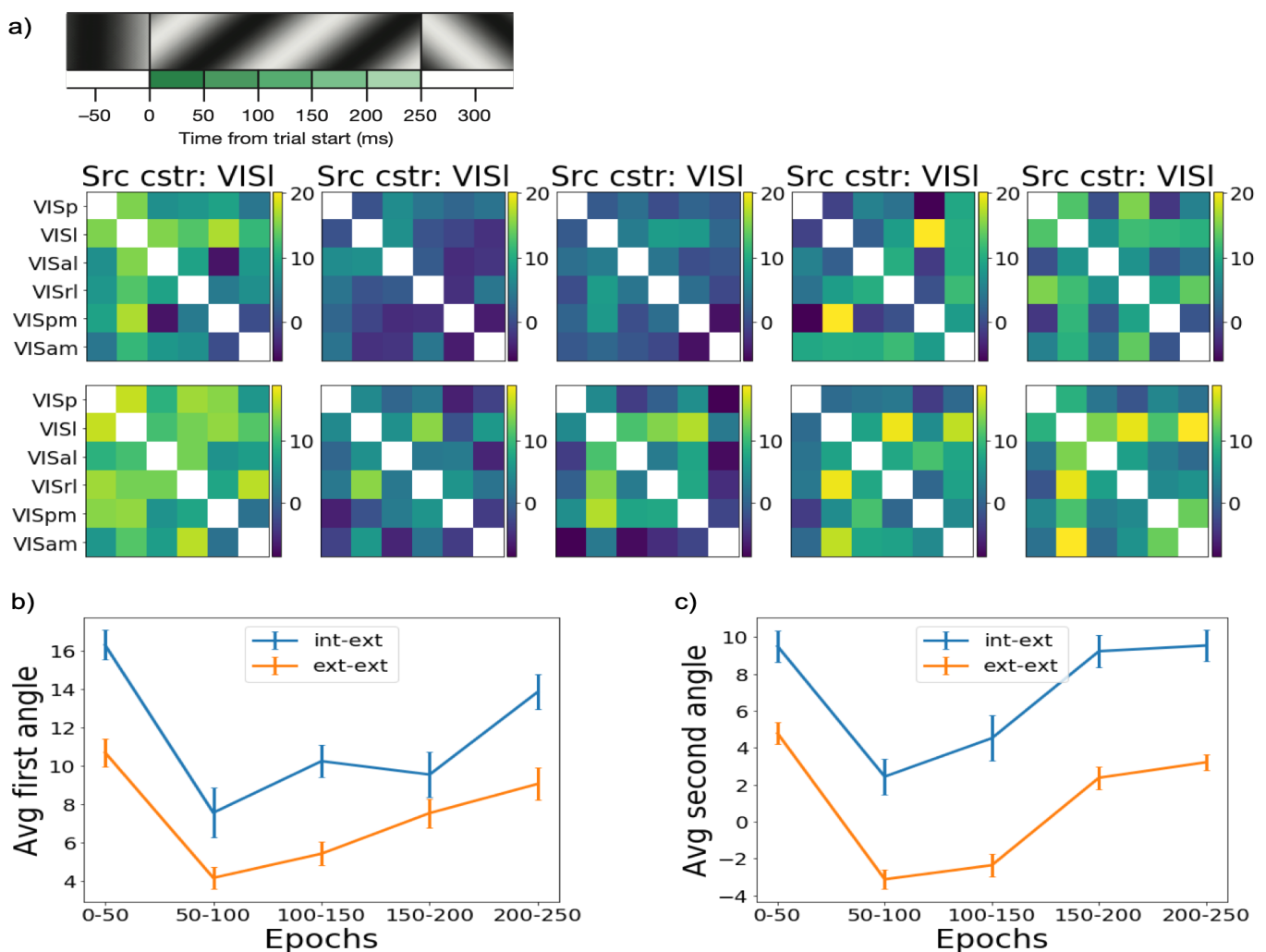

Figure S6: Subspaces rotate within single trials and become more aligned after stimulus onset and results remain unchanged even after shuffle-subtraction (see text for details). a) Top: Schematic showing division of 50ms epochs within a single trial from stimulus onset. Bottom first row: Heatmap of shuffle-subtracted first angle from a source cortical area (VISl shown here) to all possible target cortical area pairs for five successive $50 \mathrm{~ms}$ epochs (columns) within $250 \mathrm{~ms}$ trials. Bottom second row: Shuffle-subtracted second angles. Angles were computed by estimating interaction matrices using activity within each $50 \mathrm{~ms}$ epoch. Note that although subspaces become more aligned, internal-external subspace pairs are still more orthogonal than external-external subspace pairs within each epoch. b) Average of all combinations of internal-external (blue) and external-external (orange) shuffle-subtracted first angles for the five $50 \mathrm{~ms}$ epochs showing subspace alignment between 50-150 ms after stimulus onset. c) Same as in b) but for second angles.

computed subspace angles from interaction matrices obtained after shuffling activities (see Methods, model variant shuff). The same shuffle, which preserves the temporal structure of the average firing rate of the neurons was used, but it was applied to neuronal activities separated into five $50 \mathrm{~ms}$ time intervals. We applied the same method to compute the subspace angles and their relative alignment for the shuffled data, and we subtracted the shuffle control from all the angles computed.

We find that the results are unchanged when the shuffled angles are subtracted, with significant alignment between the inter- and intra-areal subspaces 50-150 ms after the stimulus onset compared to 0-50ms (Figure S6 ((Benjamini-Hochberg FDR-corrected p-values for Wilcoxon signed-rank test comparing average shuffle-subtracted angles for epochs 2-5 with epoch 1; first angle p-values : $\left(1.35 \times 10^{-13}, 3.97 \times 10^{-12}, 8.60 \times 10^{-8}, 7.50 \times 10^{-3}\right)$, second angle p-values : $\left.\left(2.18 \times 10^{-14}, 1.81 \times 10^{-12}, 1.54 \times 10^{-2}, 9.86 \times 10^{-2}\right)\right)$

\section{S3.2 Computation of subspace angles}

Directly computing the subspace angles between the matrices $\left(M_{1}^{\prime}, M_{2}^{\prime}\right)$ reconstructed using the first 10 singular vectors of $\left(M_{1}, M_{2}\right)$ did not affect our conclusions. Figure S7 (panels c) and d)) show results for both internal-external and external-external angles combining the results seen in Figures $\mathbf{S 4}$ and S5. Panel b) shows that angles obtained are higher than shuffle controls. 
bioRxiv preprint doi: https://doi.org/10.1101/2021.06.09.447638; this version posted June 23, 2021. The copyright holder for this preprint (which was not certified by peer review) is the author/funder, who has granted bioRxiv a license to display the preprint in perpetuity. It is made available under aCC-BY-NC-ND 4.0 International license.

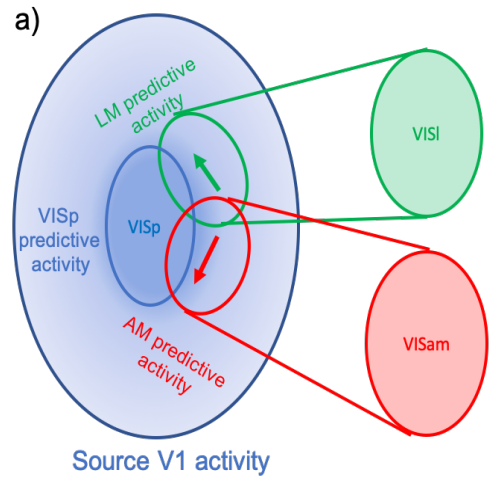

\section{Schematic of the idea}

b)

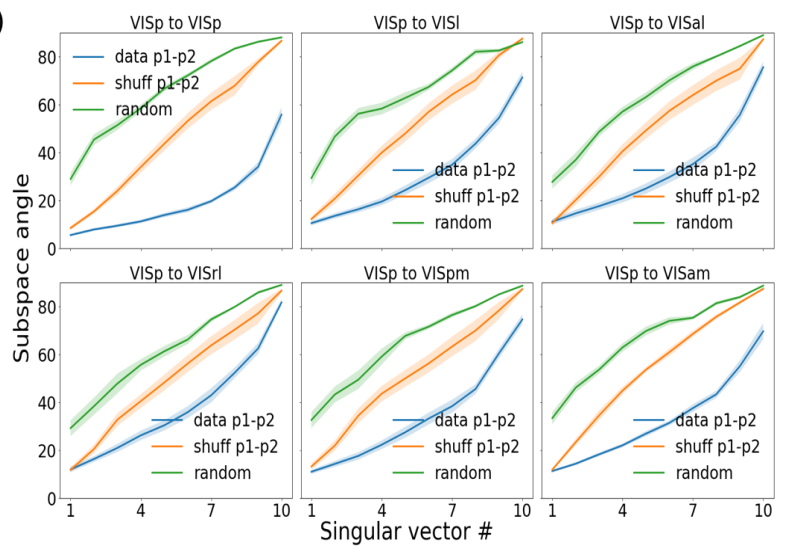

c)
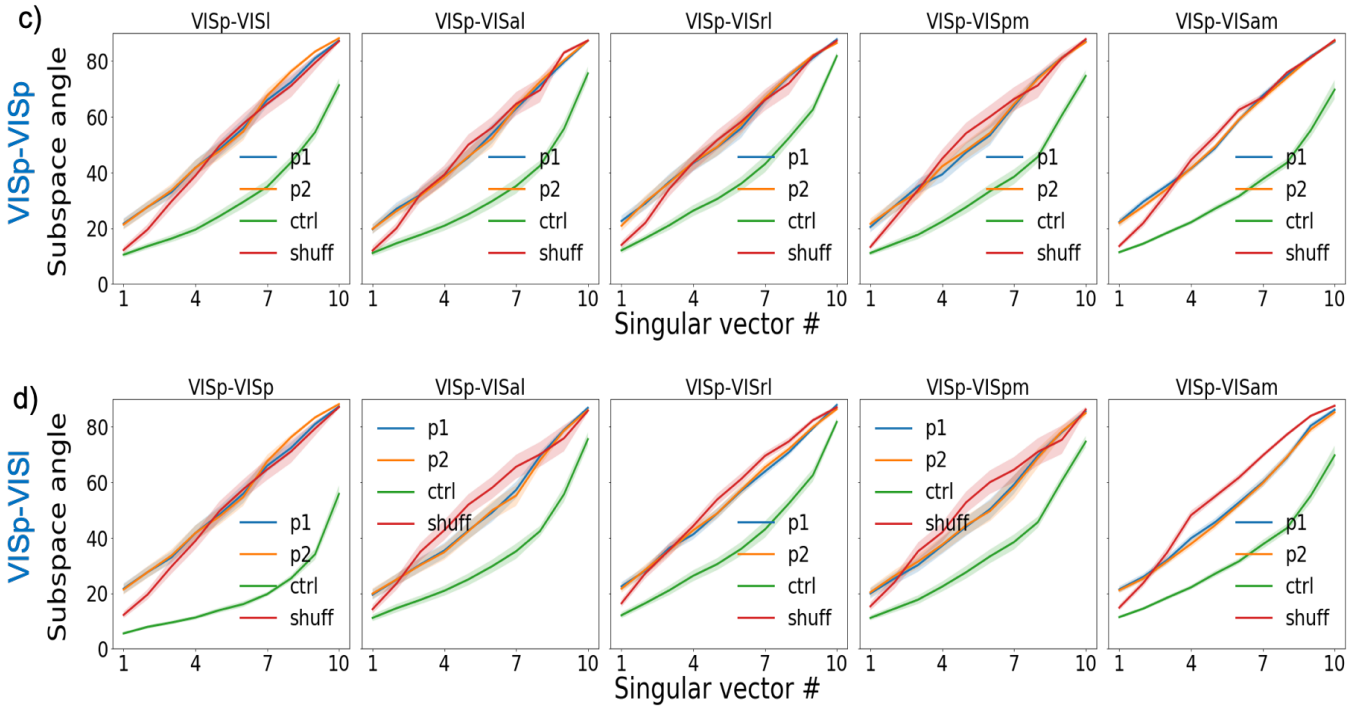

VISp-VISam

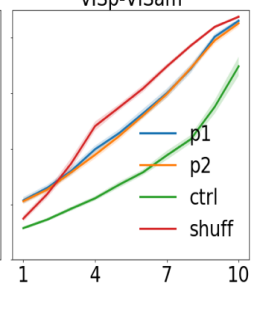

Figure S7: Subspace angles estimated directly from reconstructed interaction matrices. a) Schematic of the idea showing that distinct subspaces in VISp mediate interactions within VISp and outside with VISl. b) Subspace angles for interaction matrices from VISp to six target regions estimated using first and second halves of activity for original data (blue), shuffled data (orange) and a fully randomized condition (see Methods). Solid lines represent session averages; shaded regions show std error of mean (sem). c) Subspace angles from a fixed source region (VISp shown here) to (VISp, T) where T is one of five higher visual areas [T : VISl, VISal, VISrl, VISpm, VISam] for model variants $\mathrm{p} 1$ (blue) and p2 (orange). First few angles are significantly higher than inter-areal interaction angles (green) estimated from activities in different stimulus blocks (blue lines in panel b) as well the shuffle control (red). d) Subspace angles between VISp-VISl and (VISp, VISp) and remaining four external target subspaces estimated using first (blue) and second (orange) halves of activity for original data, shuffled data (red) and angles (green) estimated from activities in different stimulus blocks (blue lines in Fig. 2b and Fig. S4b. Solid lines represent session averages; shaded regions show std error of mean (sem).

\section{S3.3 Subspace angles from interaction matrices obtained using approach of Semedo et. al [23]}

We also computed subspace angles after estimating interaction matrices using the approach of [23]. We first computed the trial-to-trial fluctuations in response to static gratings for all sessions. We used these to relate activity fluctuations in a target area to those in a source area using reduced rank regression (RRR). To do so, we used the MATLAB code made available by the authors at (https://github.com/joao-semedo/communication-subspace). We used the mapping matrix returned by 
their code for given source and target variables as the estimated interaction matrices from which we computed the subspace angles.

Figure $\mathrm{S} 8$ shows subspace angles (averaged across all sessions) from source area $\mathrm{S}=\mathrm{VISp}$ to pairs of target areas (VISp, VISl), (VISp, VISam) and (VISl, VISam). It can be seen that the internalexternal angles are larger than the external-external angles, substantiating our result in Figure 3 that internal-external interactions are less closely aligned than external-external interactions.

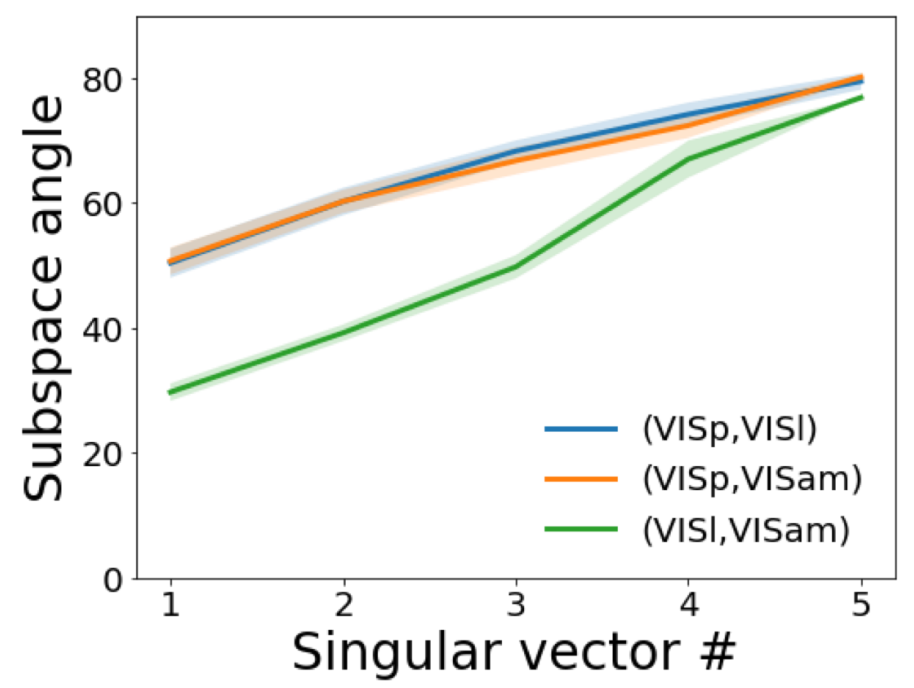

Figure S8: Subspace angles between interaction matrices estimated using reduced rank regression as outlined in [23] (see text for details) from source area S=VISp to three pairs of target areas (VISp, VISl), (VISp, VISam) and (VISl, VISam). Solid lines represent average across all sessions and shaded lines represent standard error of mean (sem). Internal-external interactions are less closely aligned than external-external interactions.

\section{S4 PredNet analysis}

We have shown that intra-areal interaction subspaces in mouse visual cortex are not well aligned with the inter-areal interaction subspaces (Figure 3). Do similar patterns of interactions exist within task-trained artificial neural networks? To allow for such a possibility, the network is likely to require interactions between neurons within an area. Therefore we analyzed interactions between the representation modules in PredNet [33, 34] - a deep convolutional recurrent neural network that was trained for next-frame video prediction. PredNet's architecture was inspired by principles of predictive coding from neuroscience [35], but it includes both feedback from higher areas as well as lateral connection on top of the feedforward connections.

We used the publicly available code-base provided by the authors of PredNet (https://github.com/coxlab/prednet). We 'probed' the activations of a subset of neurons within representation layers/modules $R_{1}, R_{3}$ and $R_{3}$ in PredNet in response to video sequences provided as test set by the authors of PredNet. The test sets consists of 83 video sequences of 10 frames each. The representation are the layers that have explicit recurrent connections. The depths of these layers are $R_{1}=48, R_{2}=96$ and $R_{3}=192$ respectively. We chose a subset of 72 neurons within each of these 3 layers by considering a $3 \times 3$ spatial region in the center and every 6 th, 12 th or 24th slices along the channel axes in these layers. We evaluated models both without and with self-interactions between units with a delay and obtained qualitatively similar results with both. For brevity, we report results here only for the model without delayed self-interactions between units. To mimic different sessions/mice as in the biological data, we split the activity matrix into 10 chunks of equal sizes and created a training set by randomly choosing 5 chunks and using the remaining chunks for testing. We repeated this process for $n=5$ random seeds from 0 to 4 inclusive and treated each such instance as a model arising from a different session. 
bioRxiv preprint doi: https://doi.org/10.1101/2021.06.09.447638; this version posted June 23, 2021. The copyright holder for this preprint (which was not certified by peer review) is the author/funder, who has granted bioRxiv a license to display the preprint in perpetuity. It is made available under aCC-BY-NC-ND 4.0 International license.

We used the peer coupling model to estimate functional interactions among the chosen neurons within and between these layers for the different model variants. Figure $\mathrm{S9}$ shows estimated interaction matrices from source module $R_{2}$ to target modules $R_{1}$ and $R_{2}$.

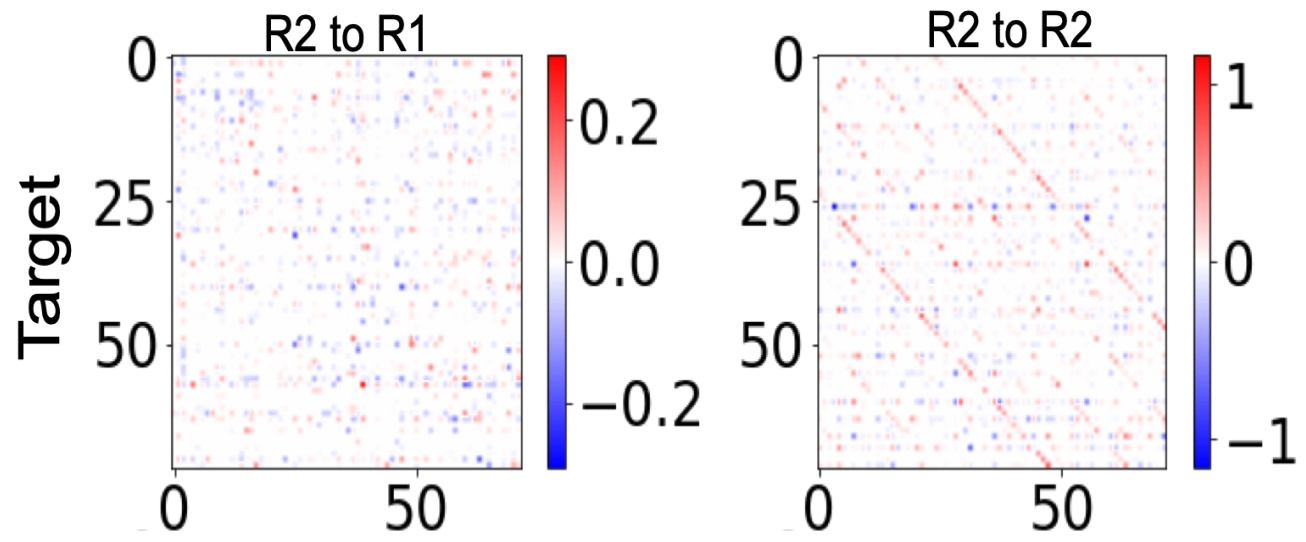

Source

Figure S9: Functional interactions in PredNet obtained with our model for source module $R_{2}$ to target modules $R_{1}$ (left) and $R_{2}$ (right) respectively. Model does not include delayed self-interactions for units (see text for details).

In contrast to the biological network, our analysis did not result in significant differences between internal-external and external-external interactions within PredNet (Figure S10|(Benjamini-Hochberg FDR-corrected p-values for the Mann-Whitney U test for the three representation modules respectively: $(0.613,0.860,0.275)$ for the first angles and $(0.987,0.306,0.404)$ for the second angles))
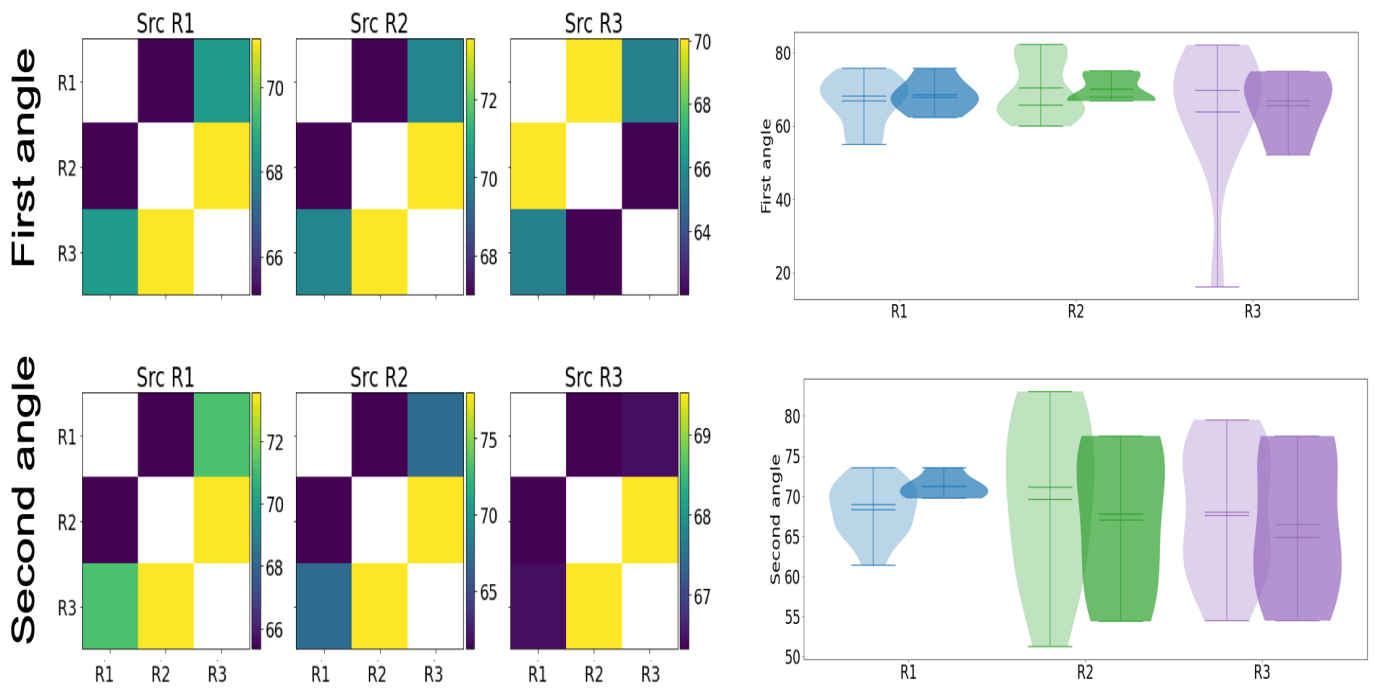

Figure S10: Internal-external and external-external interactions within PredNet are not significantly different from each other. Left: Heatmaps show average first angle (top row) and second angle (bottom row) across different random seeds that act as a proxy for different sessions/mice (see text for details). Right: Distributions of internal-external and external-external angles (top; first angle, bottom; second angle). Each pair of violinplots with different shades of the same color represents internal-external (light shade) and external-external (dark shade) angles respectively. 
To investigate if noise influences the geometry of interactions, we injected a small amount of noise in to the activities of chosen units in each module and then re-computed subspace angles after estimating the respective interaction matrices. For each module, we added Gaussian white noise to all activities within that module with zero mean and standard deviation $\sigma_{s}=0.01 \times \sigma$, where $\sigma$ is the standard deviation of activities of the chosen 72 units within that module. Again, we did not find significant differences in internal-external and external-external interactions between PredNet modules (Figure S11) (Benjamini-Hochberg FDR-corrected p-values for the Mann-Whitney $\mathrm{U}$ test for the three representation modules respectively: $(0.403,0.370,0.879)$ for the first angles and $(0.403,0.306,0.840)$ for the second angles)).
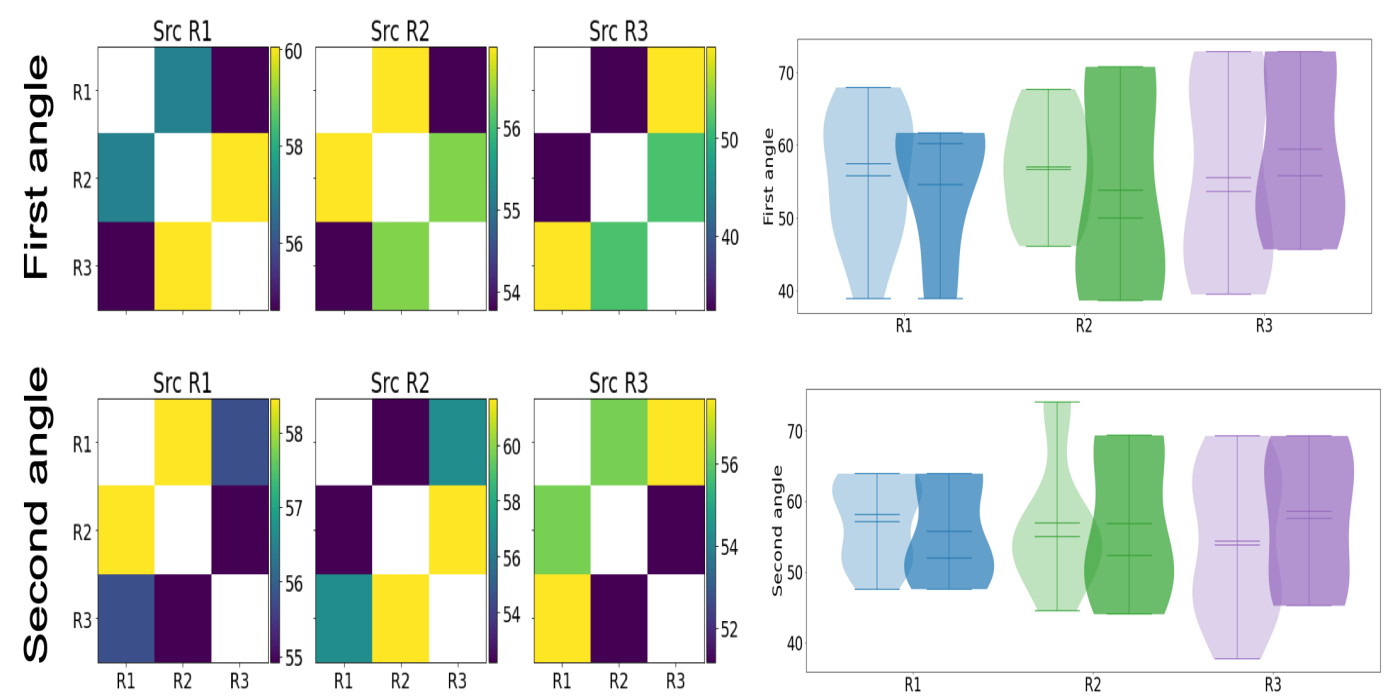

Figure S11: Internal-external and external-external interactions within PredNet are not significantly different from each other even after adding Gaussian white noise to chosen unit activities within respective modules (see text for details). Left: Heatmaps show average first angle (top row) and second angle (bottom row) across different random seeds that act as a proxy for different sessions/mice (see text for details). Right: Distributions of internal-external and external-external angles (top; first angle, bottom; second angle). Each pair of violinplots with different shades of the same color represents internal-external (light shade) and external-external (dark shade) angles respectively.

In PredNet, the absence of significant differences in internal-external and external-external interactions is similar to what is observed in mouse visual cortical areas 50-100 ms after stimulus onset when the misalignment between subspaces disappears (Figure 4 and Figure $\mathbf{S 6}$ ). There are many possible reasons for the observed differences in interaction patterns in PredNet and visual cortical areas and we can only speculate. One possibility is that visual cortical areas can dynamically rotate the interaction subspaces and reduce the misalignment only when task relevant, while in the task-driven PredNet network the representation modules with recurrent interactions do not become misaligned. 